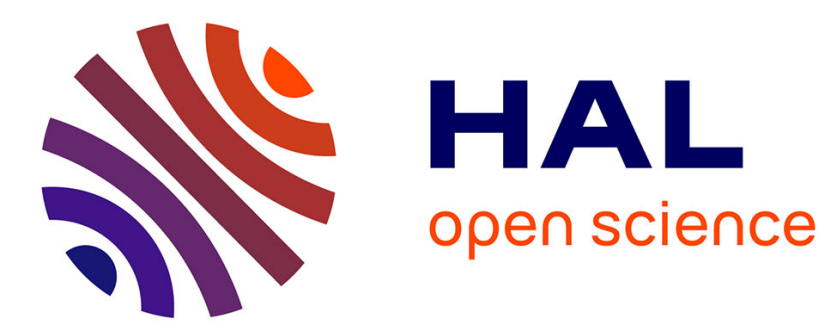

\title{
Forward and Backward Dynamics in implicitly defined Overl apping Generations Models
}

\author{
Laura Gardini, Cars Hommes, Fabio Tramontana, Robin de Vilder
}

\section{To cite this version:}

Laura Gardini, Cars Hommes, Fabio Tramontana, Robin de Vilder. Forward and Backward Dynamics in implicitly defined Overl apping Generations Models. Journal of Economic Behavior and Organization, 2009, 71 (2), pp.110. 10.1016/j.jebo.2008.09.010 . hal-00693817

\section{HAL Id: hal-00693817 https://hal.science/hal-00693817}

Submitted on 3 May 2012

HAL is a multi-disciplinary open access archive for the deposit and dissemination of scientific research documents, whether they are published or not. The documents may come from teaching and research institutions in France or abroad, or from public or private research centers.
L'archive ouverte pluridisciplinaire HAL, est destinée au dépôt et à la diffusion de documents scientifiques de niveau recherche, publiés ou non, émanant des établissements d'enseignement et de recherche français ou étrangers, des laboratoires publics ou privés. 


\section{Accepted Manuscript}

Title: Forward and Backward Dynamics in implicitly defined Overl apping Generations Models

Authors: Laura Gardini, Cars Hommes, Fabio Tramontana, Robin de Vilder

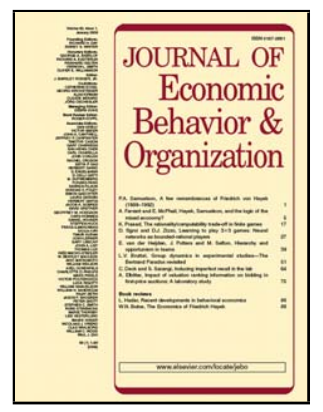

PII: S0167-2681(09)00106-1

DOI: doi:10.1016/j.jebo.2008.09.010

Reference: JEBO 2365

To appear in: Journal of Economic Behavior \& Organization

Received date: $\quad$ 14-7-2004

Accepted date: $\quad 3-9-2008$

Please cite this article as: Gardini, L., Hommes, C., Tramontana, F., de Vilder, R., Forward and Backward Dynamics in implicitly defined Overl apping Generations Models, Journal of Economic Behavior and Organization (2008), doi:10.1016/j.jebo.2008.09.010

This is a PDF file of an unedited manuscript that has been accepted for publication. As a service to our customers we are providing this early version of the manuscript. The manuscript will undergo copyediting, typesetting, and review of the resulting proof before it is published in its final form. Please note that during the production process errors may be discovered which could affect the content, and all legal disclaimers that apply to the journal pertain. 


\title{
Forward and Backward Dynamics in implicitly defined Overlapping Generations Models*
}

\author{
Laura Gardini ${ }^{a}$, Cars Hommes ${ }^{b}$, Fabio Tramontana ${ }^{c}$, Robin \\ de Vilder $^{d}$
}

\begin{abstract}
In dynamic economic models derived from optimization principles, the forward equilibrium dynamics may not be uniquely defined, while the backward dynamics is well defined. We derive properties of the global forward equilibrium paths based on properties of the backward dynamics. We propose the framework of iterated function systems (IFS) to describe the set of forward equilibria and apply the IFS framework to a one- and a two-dimensional version of the overlapping generations (OLG)-model. We show that if the backward dynamics is chaotic and has a homoclinic orbit (a "snap-back repeller"), the set of forward equilibrium paths converges to a fractal attractor. Forward equilibria may be interpreted as sunspot equilibria, where a random sunspot sequence determines equilibrium selection at each date.
\end{abstract}

Key words: forward and backward dynamics, chaos, homoclinic orbit, snap-back repellor, sunspot equilibria, overlapping generations models.

JEL classification: E32, C62, C02

* This paper has a long history. An earlier version under the title "Sunspot equilibria in an implicitly defined overlapping generations model" by two authors ( $\mathrm{CH}$ and RdeV) appeared as CEPREMAP Working Paper 1995 and already contained the main intuition. Two additional authors (LG and FT) provided a rigorous mathematical framework to support this intuition. We would like to thank Jean-Michel Grandmont, Sebastian van Strien, Duncan Sands, Frank Portier, Pierre Malgrange, Patrick Pintus and Alfredo Medio for many stimulating discussions and very useful remarks and suggestions. We are also indebted to four anonymous referees for their detailed comments on an earlier draft and many suggestions for improvements, and to the Editor, Barkley Rosser Jr., for his encouragement to finish this project. All errors that remain after so many years are entirely ours.

${ }^{a}$ Department of Economics, University of Urbino, Italy.

${ }^{b}$ CeNDEF, Department of Quantitative Economics, University of Amsterdam

${ }^{c}$ Department of Economics, University of Ancona, Italy.

${ }^{d}$ Department of Mathematics, University of Amsterdam. 


\section{Introduction.}

Dynamic economic models based on optimization principles are often described by implicitly defined equilibrium equations derived from first order conditions. In such an implicitly defined system, the forward equilibrium dynamics is in general not uniquely defined, since at each date there may be more than one optimal forward solution, while the backward dynamics may be well-defined. This situation typically occurs if the dynamic equations are nonlinear, non-invertible functions (e.g. power functions). Two well known examples are the overlapping generations (OLG)-model (e.g. Grandmont, 1985) and the cash-in-advance model (e.g. Woodford, 1994, Michener and Ravikumar, 1998).

One way to deal with this ambiguity is to select the solution closest to the steady state. Indeed locally this strategy makes sense in a dynamic analysis. However, globally there often is no inherent reason, other than convenience, why one should follow this strategy. At first sight it seems unwise to select a far from steady state solution; the dynamical analysis would get unnecessarily complicated and presumably not much can be said about the global forward dynamics. The aim of this paper is to characterize the non-uniquely defined forward equilibrium paths using knowledge of the well-defined backward dynamics. We present examples of the OLG-model in which at each date multiple forward equilibria exist, but all forward equilibrium paths converge to a fractal attractor.

This seemingly contradictory claim can most easily be understood by means of an example. Take three arbitrary linear contracting maps $f_{i}(i=1,2,3)$ of the plane. If one applies one map, say $f_{1}$ sufficiently often then sequences will converge to the unique fixed point of $f_{1}$. A similar conclusion holds if one uses one of the other two maps. The picture dramatically changes if one allows for a random choice between the three maps at each period in time. More precisely, it has been shown by Barnsley (1988) that independent of the starting condition and the random mechanism that prescribes the choice of maps all orbits converge to an unique fractal attractor. The dynamics on the attractor displays extremely irregular though unique behaviour: two different initial conditions will converge rather than diverge to the same unique, irregular dynamic path.

This remarkable result is just a simple example of a so-called iterated functions system (IFS). An IFS provides a natural framework to describe the global equilibrium dynamics in implicitly, non-uniquely defined dynamic equilibrium models. To see this consider the nonlinear pure exchange overlapping generations (OLG) model thoroughly analyzed by Grandmont (1985). In his seminal paper Grandmont shows that in the Samuelson case the "forward" equilibrium dynamics may 
not be uniquely defined due to a strong conflict between substitution and income effects. That is, in order to obtain the perfect foresight equilibrium dynamics one has to invert a hump shaped offer curve. To deal with this problem Grandmont (1985) first analyses the "backward" dynamics and subsequently translates the backward dynamical phenomena through a learning mechanism to the forward process (see also Grandmont and Laroque, 1986). We follow a different strategy: we call the part of the offer curve that lies to the left (right) of the critical value the left (right) forward map. In this way we obtain two invertible functions, one monotonically increasing and the other monotonically decreasing, each map having a unique attracting fixed point. Using the theory of IFS, with some slight modifications, we investigate the forward equilibrium dynamics of the model. Our method captures all possible forward equilibrium paths, including those shown to exist by Grandmont (1985). In particular, we will show that the forward equilibrium paths converge to a Cantor set, typically jumping erratically over the Cantor set.

We also apply the (modified) theory of IFS to a non-invertible twodimensional OLG model with productive investment and capital accumulation and a fixed factor production technology. In the case the twodimensional model is invertible and agents have perfect foresight it has been shown that purely deterministic periodic (Grandmont, 1993 and Reichlin, 1986) as well as chaotic (de Vilder 1995, 1996) equilibrium paths are possible outcomes, even when the two goods are gross substitutes. Moreover, Woodford (1986) showed, in an infinite horizon interpretation of the model, the existence of sunspot equilibria (generated by stochastic shocks to expectations) near the stable monetary steady state $^{1}$. Here we apply the IFS framework to the two-dimensional OLGmodel when the offer curve is non-monotonic due to a sufficiently strong income effect.

In both the one-and two-dimensional OLG model agents have rational expectations. The forward rational expectations equilibrium paths can be interpreted as sunspot equilibria, where a random sunspot sequence determines equilibrium selection, that is, at each date a random sunspot sequence determines which of the multiple equilibria is selected. See Azariadis and Guesnerie (1986), Boldrin and Montrucchio (1986), Cass and Shell (1983), Grandmont (1993) or Woodford $(1984,1986)$ for

\footnotetext{
${ }^{1}$ Grandmont et al. (1998) and Pintus et al. (2000) generalized these results by showing that sunspot equilibria and regular and irregular purely deterministic phenomena also arise when the production process allows for factor substitution. See also Cazzavillan et al. (1998), who allow for increasing returns to scale in various versions of the twodimensional model.
} 
general references on the sunspot literature.

There have been a number of related approaches to describe forward dynamics in implicitly defined systems. Kamihigashi (1999) describes the set of equilibria in implicitly defined equilibrium models by symbolic dynamics and shows the existence of chaotic equilibrium paths. Contrary to our approach, Kamihigashi does not investigate whether such chaotic equilibria can be attracting and also does not use the notion of IFS. More recently, Medio and Raines (2007) use the theory of "inverse limits" to analyze the long run dynamical behaviour of implicitly defined OLG-models. Even though the forward dynamics are multi-valued, they show that typical long run equilibria correspond to an attractor of the shift map on the inverse limit space. In a similar vein Kennedy and Stockman (2008) apply the theory of inverse limits to both cash-in-advance and OLG models and provide necessary and sufficient conditions for these systems to be chaotic. See also the recent discussion in Grandmont (2008). A problem with the inverse limit approach however, is that it is rather abstract and, e.g., the inverse limit space is infinitely dimensional. Our IFS approach is much simpler and intuitive. We present sufficient conditions, which can be verified graphically or numerically, on the (uniquely defined) backward map for the forward dynamics to be chaotic. We also present intuitive ways to simulate the forward dynamics, by randomly (possibly with some restrictions) applying one of the inverse forward maps, generating sunspot equilibria corresponding to a fractal attractor of some suitable IFS.

We have organized the paper as follows. Section 2 briefly recalls the OLG-model, defines the backward and foreward maps and discusses sunspot equilibria. In section 3 we present the formalism of iterated function systems (IFS), mainly by means of simple examples. Sections 4 and 5 apply this formalism to the one-respectively two-dimensional OLG-model. Finally, some concluding remarks are given in section 6 and a mathematical appendix recalls some basic notions from dynamical systems theory.

\section{The Overlapping Generations models}

In this section we present both a one-and a two-dimensional overlapping generations (OLG-)models. We start with the simpler one-dimensional model, followed by the closely related two-dimensional model.

\subsection{One-dimensional OLG-model}

Consider the OLG-model as in Grandmont (1985, 1993). The economy has a constant population and is built up from a household sector and a production sector. Both sectors have a representative agent who lives for 
two periods and behaves rationally. The representative agent from the household sector works when young and consumes when old. In return for their effort the young receive a salary in the form of fiat money which is available in a fixed amount. The consumption good is assumed to be perishable and one unit is produced from one unit of labour supplied by the young. Agents have a separable utility function $V_{1}\left(l^{*}-l_{t}\right)+V_{2}\left(c_{t+1}^{e}\right)$, where $l^{*}$ is fixed labour endowment of the young, $l^{*}-l_{t}$ is current leisure and $c_{t+1}^{e}$ is next period's expected consumption. Agents optimize their utility under the budget constraint $w_{t} l_{t}=p_{t+1}^{e} c_{t+1}^{e}=M$, where $w_{t}$ is wage rate, $p_{t+1}^{e}$ expected price of the consumption good in period $t+1$ and $M$ denotes fiat money. Under perfect foresight, the first order condition is $v_{1}\left(l_{t}\right)=l_{t} V_{1}^{\prime}\left(l^{*}-l_{t}\right)=c_{t+1} V_{2}^{\prime}\left(c_{t+1}\right)=v_{2}\left(c_{t+1}\right)$. The optimal pair of current labour and future consumption is then given by

$$
l_{t}=\chi\left(c_{t+1}\right)=v_{1}^{-1} \circ v_{2}\left(c_{t+1}\right),
$$

with $\chi()=.v_{1}^{-1} \circ v_{2}($.$) ; its graph is the so-called offercurve. Since one$ unit of labor produces one unit of consumption good we have output $y_{t}=l_{t}=c_{t}$, so that we obtain

$$
y_{t}=\chi\left(y_{t+1}\right)
$$

We will refer to (2) as the backward dynamics. Note that the backward dynamics is uniquely defined, since $\chi=v_{1}^{-1} \circ v_{2}$ and $v_{1}(l)=l V_{1}^{\prime}\left(l^{*}-l\right)$ is increasing for a concave utility function. To study the forward dynamics of this one-dimensional OLG economy one has to invert the function $\chi$ to obtain:

$$
y_{t+1}=\chi^{-1}\left(y_{t}\right) .
$$

We will refer to (3) as the forward dynamics. The forward dynamics is only uniquely defined if $v_{2}^{-1}$ is well-defined, that is, if $=v_{2}\left(c_{t+1}\right)=$ $c_{t+1} V_{2}{ }^{\prime}\left(c_{t+1}\right)$ is monotonic. This is the case if current leisure and future consumption are gross substitutes, and in that case the one-dimensional economy described by (3) can only display simple dynamic behavior: convergence to a steady state equilibrium (see e.g. Kehoe et al., 1991).

In his seminal paper Grandmont (1985) has shown that, if the two goods are not gross substitutes and the income effect is sufficiently strong, the offercurve is backward bending and non-monotonic. When the income effect is strong, the backward dynamics (2) may exhibit complicated, chaotic fluctuations. This result heavily relies on a well known theorem of Sharkovsky (1964) (see also Sharkovsky et al. 1997) and $\mathrm{Li}$ and Yorke (1975), which essentially says that if a continuous one-dimensional dynamical system has a periodic orbit of period three then there exist infinitely many coexisting periodic orbits as well as an 
uncountable set of aperiodic orbits all living in a Cantor set, usually of Lebesgue measure zero ${ }^{2}$. In order to translate these results to the forward equilibrium process ${ }^{3}$ Grandmont (1985) introduces a learning mechanism, and shows that a backward unstable periodic orbit (or cycle) is locally a stable cycle in the forward equilibrium process with learning (i.e., for a specific sequence of inverses).

When the offercurve is non-monotonic, the forward dynamics (3) is not uniquely defined, and it is better to speak of a forward process. Our main concern here will be: what can we say about the equilibrium paths of the forward process, given our knowledge of the backward dynamics. We will, for example, see that if the backward dynamics exhibits chaotic dynamics, then the forward process will also exhibit complicated dynamical behavior.

\subsection{Two-dimensional OLG-model}

We now introduce the two-dimensional OLG-model considered in Reichlin (1986), Woodford (1986) and Grandmont (1993). There are two sectors in the economy, a household sector and a production sector. The household sector is the same as in the one-dimensional OLG-model discussed above. Hence, as before, under perfect foresight the optimal pair of current labour and future consumption is given by

$$
l_{t}=\chi\left(c_{t+1}\right),
$$

with $\chi$ the offercurve. Solving for future consumption yields

$$
c_{t+1}=\chi^{-1}\left(l_{t}\right) .
$$

Output is not only produced from labour $l_{t}$ supplied by the household sector, but also from capital stock $k_{t-1}$ supplied by non-consuming, profit maximizing entrepreneurs. Both inputs are being used in fixed proportions, that is, we assume a Leontief production technology so that output $y_{t}=\min \left\{l_{t}, k_{t-1} / a\right\}$, where $1 / a$ is the productivity of capital. In each period output is partly directed towards the household sector for consumption and partly directed towards the capital sector for investment. The capital stock available for production, at the beginning of period $t+1$, is:

$$
k_{t}=(1-\delta) k_{t-1}+i_{t}, \quad 0<\delta<1,
$$

\footnotetext{
${ }^{2}$ We suggest the interested reader to consult the treatments of Grandmont $(1985,1988)$ or Devaney (1986).

${ }^{3}$ In the sequel we sometimes just refer to the forward equilibrium dynamics.
} 
where $\delta$ is the depreciation rate of capital and $i_{t}$ is investment.

In equilibrium $y_{t}=l_{t}=k_{t-1} / a, c_{t}=y_{t}-i_{t}$ and, using (6), investment can be written as

$$
i_{t}=a\left[y_{t+1}-y_{t}(1-\delta)\right]
$$

Actual future output is then given by

$$
y_{t+1}=(1-\delta) y_{t}+\frac{1}{a}\left(y_{t}-c_{t}\right)
$$

Moving one period forward yields

$$
y_{t+2}=\left(1-\delta+\frac{1}{a}\right) y_{t+1}-\frac{1}{a} c_{t+1} .
$$

Using $c_{t+1}=\chi^{-1}\left(l_{t}\right)$ and $l_{t}=y_{t}$ we get

$$
y_{t+2}=\left(1-\delta+\frac{1}{a}\right) y_{t+1}-\frac{1}{a} \chi^{-1}\left(y_{t}\right) .
$$

Substituting $q_{t}=y_{t}$ and $r_{t}=y_{t+1}$ yields the two-dimensional system

$$
\begin{aligned}
& q_{t+1}=r_{t} \\
& r_{t+1}=\left(1-\delta+\frac{1}{a}\right) r_{t}-\frac{1}{a} \chi^{-1}\left(q_{t}\right) .
\end{aligned}
$$

Equations (11)-(12) define the forward dynamics of the 2-D OLG-model. As in the 1-D case, the forward dynamics is only uniquely defined if the offercurve is monotonic, so that the inverse $\chi^{-1}$ is uniquely defined. If the offercurve is non-monotonic, the forward process (11)-(12) is not uniquely defined and at each date $t$ there are two possibilities for continuation depending on whether one applies the left inverse $\chi_{L}^{-1}$ or the right inverse $\chi_{R}^{-1}$. It is straightforward to use (11)-(12) to derive the backward dynamics:

$$
\begin{aligned}
q_{t} & =\chi\left[a\left(1-\delta+\frac{1}{a}\right) q_{t+1}-a r_{t+1}\right] \\
r_{t} & =q_{t+1} .
\end{aligned}
$$

As in the 1-D OLG-model, the backward dynamics is always well-defined, and we can formulate the same problem for the 2-D OLG-model: what can we say about the equilibrium paths of the forward process (11)-(12), given our knowledge of the backward dynamics (13)-(14)? As in the 1-D case, we will see that if the backward dynamics exhibits chaotic dynamics, then the forward process will also exhibit complicated dynamical behavior. 


\subsection{Sunspot equilibria}

In this subsection we discuss the relation between perfect foresight equilibria and sunspot equilibria. In general the relationship between sunspot equilibria and deterministic dynamics is complicated (see e.g. Cass and Shell, 1983, Azariadis and Guesnerie, 1986, Grandmont, 1993 and Woodford, 1986, for a general discussion of sunspot equilibria), but in our setting, there is a simple relationship when a random sunspot sequence serves as an equilibrium selection process ${ }^{4}$.

First consider the simpler 1-D OLG model. At date $t$, given the current money wage $w_{t}>0$ and expectations about (possibly random) future prices $p_{t+1}^{e}$, consumers maximize expected utility:

$$
V_{1}\left(l^{*}-l_{t}\right)+E_{t} V_{2}\left(c_{t+1}^{e}\right),
$$

where $E_{t}$ denotes conditional mathematical expectations of a random variable, subject to the (possibly random) budget constraint $w_{t} l_{t}=$ $p_{t+1}^{e} c_{t+1}^{e}$. The first order condition yields

$$
v_{1}\left(l_{t}\right)=E_{t} v_{2}\left(c_{t+1}^{e}\right),
$$

which together with the budget constraint determines (uniquely) the current labour supply $l_{t}$ and (possibly random) expected consumption $c_{t+1}^{e}$ as a function of $w_{t}$ and expected future price $p_{t+1}^{e}$.

In the 1-D OLG-model, where $w_{t}=p_{t}$ and $y_{t}=c_{t}=l_{t}=M / p_{t}$, a stochastic rational expectations equilibrium is described by a sequence of random variables $y_{t}>0$, for $t \geq 0$, satisfying $v_{1}\left(y_{t}\right)=E_{t} v_{2}\left(y_{t+1}\right)$. This is a sunspot equilibrium in the sense that it can be interpreted as an equilibrium where agents believe (rationally) that equilibrium prices $p_{t}=M / y_{t}$ are correlated with a stochastic sunspot sequence $s_{t}, t=1,2,3, \cdots$ that do not affect economic "fundamentals". A particular case is a deterministic perfect foresight equilibrium: a sequence of deterministic outputs $y_{t}, t \geq 0$, satisfying $v_{1}\left(y_{t}\right)=v_{2}\left(y_{t+1}\right)$ or $y_{t}=\chi\left(y_{t+1}\right)$. In our setting, the sunspot process only performs a random selection at time $t+1$ between the two deterministic equilibria $y_{t+1}=M / p_{t+1}$ that are possible continuations, given $y_{t}=M / p_{t}$, at date $t$. More precisely, given $p_{t}=M / y_{t}$, let $p_{t+1, i}=M / y_{t+1, i}$ be such that $y_{t}=\chi\left(y_{t+1, i}\right)$ or $v_{1}\left(y_{t}\right)=v_{2}\left(y_{t+1, i}\right)$, for $i=1,2$. Suppose that $p_{t+1, i}$ is selected (perhaps through the realization of some sunspot variable $s_{t+1, i}$ ) with probability $q_{i} \geq 0$. Then at date $t$, the relation $v_{1}\left(y_{t}\right)=E_{t}\left(v_{2}\left(y_{t+1}\right)\right)=\sum_{i} q_{i} v_{2}\left(y_{t+1, i}\right)$ in (16) is satisfied. The random selection that will be performed at date $t+1$ does not affect the choice of households at date $t$, hence the equilibrium

\footnotetext{
${ }^{4}$ We would like to thank an anonymous referee for spelling out the details of this relationship.
} 
price $p_{t}$. Hence, if sunspots only perform at each date a random selection between the two possible deterministic temporary perfect foresight equilibria, given past realizations, then the sample trajectories are forward perfect foresight equilibria generated by (3). Notice that, at each date, the random sunspot sequence in fact only must prescribe whether the left inverse $\chi_{L}^{-1}$ or the right inverse $\chi_{R}^{-1}$ is applied to select the next equilibrium in (3).

In the 2-D OLG-model a similar relation with sunspot equilibria arises. Sunspot equilibria in the 2-D OLG model with Leontief technology satisfy

$$
v_{1}\left(y_{t}\right)=E_{t} V_{2}\left[(1+a(1-\delta)) y_{t+1}-a y_{t+2}\right] .
$$

Note that, since $y_{t+1}=k_{t} / a$ is predetermined at date $t+1$, only $y_{t+2}=$ $k_{t+1} / a$ is random here, given information available at date $t$. Deterministic perfect foresight equilibria satisfy

$$
y_{t}=\chi\left[(1+a(1-\delta)) y_{t+1}-a y_{t+2}\right] .
$$

derived from (10). The same argument as in the 1-D OLG-model then shows that stochastic equilibria where sunspots only perform a random selection between the two perfect foresight equilibria at date $t+1$, given the past (i.e. between values of $y_{t+2}$ satisfying (10) given $y_{t+1}$ and $y_{t}$ ) generate sample paths that are forward perfect foresight equilibria. As in the 1-D case, at each date, the random sunspot sequence only must prescribe whether the left inverse $\chi_{L}^{-1}$ or the right inverse $\chi_{R}^{-1}$ is applied to select the next equilibrium in (11)-(12).

\section{Iterated Function System (IFS)}

The theory of so-called iterated function systems (IFS) provides a natural framework to deal with implicitly, non-uniquely defined equilibrium models. Since most economists will not be familiar with the notion of IFS we introduce in this section its main characteristics by means of examples and formalize the essential features afterwards. For a detailed treatment of the theory of IFS see Barnsley and Demko (1985) and Barnsley (1988).

\subsection{An attracting Cantor set}

Our starting point is the definition of a "Cantor set", which plays an important role as a repelling invariant set in chaotic systems and as a fractal attractor of an IFS. The Mathematical Appendix briefly recalls some basic notions from dynamical systems, such as invariant sets, repellors and attractors. We recall that by definition (see e.g. Devaney 1986), a set $\Lambda$ is a Cantor set if it is closed, totally disconnected and per- 
fect $^{5}$. The simplest example is the "Middle-third Cantor set": start with a closed interval I and remove the open "middle third" of the interval (see Figure 1). Next, from each of the two remaining closed intervals, say $I_{0}$ and $I_{1}$, remove again the open "middle thirds", and so on. After $n$ iterations, we have $2^{n}$ closed intervals inside the two intervals $I_{0}$ and $I_{1}$.

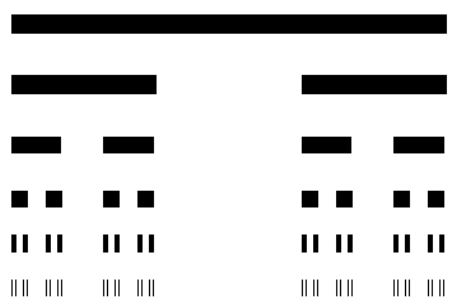

Figure 1: Middle-third Cantor set

Cantor sets are obtained in a natural way as chaotic invariant sets in dynamical systems, see e.g. Devaney (1986) or Li and Yorke (1975). To illustrate this point, consider the well known logistic map ${ }^{6}$

$$
x_{t}=f_{\mu}\left(x_{t+1}\right)=\mu x_{t+1}\left(1-x_{t+1}\right) .
$$

Notice that we have written this quadratic difference equation as a welldefined backward dynamical system, as for the 1-D OLG-model in 2. Since $f_{\mu}$ is non-monotonic, its inverse map is not unique. For any point $\xi$ belonging to the interval $I=[0,1]$ there are two distinct inverse functions, say $f_{\mu}^{-1}(\xi)=f_{0}^{-1}(\xi) \cup f_{1}^{-1}(\xi)$, where ${ }^{7}$.

$$
f_{0}^{-1}(\xi)=\frac{\mu-\sqrt{\mu^{2}-4 \mu \xi}}{2 \mu} \quad, \quad f_{1}^{-1}(\xi)=\frac{\mu+\sqrt{\mu^{2}-4 \mu \xi}}{2 \mu} .
$$

Hence, as for the 1-D OLG-model, the forward dynamics is not uniquely defined. We will show that for $\mu>2+\sqrt{5}$, the backward dynamics (19) has chaotic invariant Cantor set $\Lambda^{8}$. Moreover, we will show that the same Cantor set is an attractor of the so-called iterated function system formed by the two inverses $f_{0}^{-1}(\xi)$ and $f_{1}^{-1}(\xi)$. A first observation is that for $\mu>2+\sqrt{5}$ the two inverses $f_{0}^{-1}(\xi)$ and $f_{1}^{-1}(\xi)$ are contractions in I.

${ }^{5}$ Totally disconnected means that it contains no intervals (i.e. no subset $[a, b]$ with $a \neq b$ ) and perfect means that every point is a limit point of other points of the set.

${ }^{6}$ This example was used within the theory of OLG-models by Medio and Raines (2007).

${ }^{7}$ Instead of the two symbols 0 and 1 , one may use $L$ and $R$ respectively.

${ }^{8}$ In fact, this is true for any $\mu>4$, but the proof is more complex. 


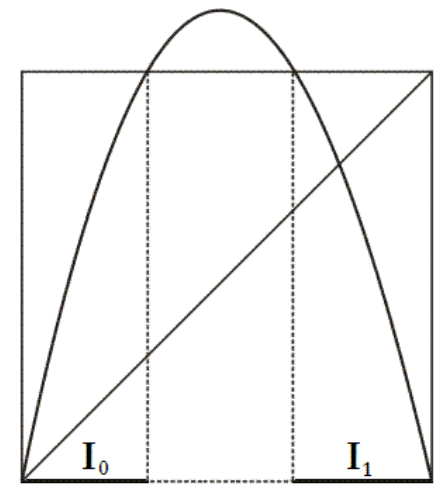

Figure 2: Logistic map for $\mu>2+\sqrt{5}$ and the two disjoint intervals $f_{\mu}^{-1}(I)=I_{0} \cup I_{1}$.

The set of points whose backward dynamics is bounded forever in the interval $I$ can be obtained by removing from the interval all the points which exit the interval after $n$ iterations, for $n=1,2, \ldots$. Thus let us start with the two closed disjoint intervals

$$
f_{\mu}^{-1}(I)=f_{0}^{-1}(I) \cup f_{1}^{-1}(I)=I_{0} \cup I_{1},
$$

(see Figure 2), i.e. we have removed the points leaving $I$ after one backward iteration. Next we remove the points exiting after two backward iterations obtaining four closed disjoint intervals

$$
f_{\mu}^{-2}(I)=I_{00} \cup I_{10} \cup I_{01} \cup I_{11},
$$

defining in a natural way $f_{\mu}^{-1}\left(I_{0}\right)=f_{0}^{-1}\left(I_{0}\right) \cup f_{1}^{-1}\left(I_{0}\right)=I_{00} \cup I_{10}$ and $f_{\mu}^{-1}\left(I_{1}\right)=f_{0}^{-1}\left(I_{1}\right) \cup f_{1}^{-1}\left(I_{1}\right)=I_{01} \cup I_{11}$. Note that if a point $x$ belongs to $I_{01}$ (or to $I_{11}$ ) then $f_{\mu}(x)$ belongs to $I_{1}$ (i.e. one iteration means dropping the first symbol of the index). Continuing the elimination process we have that $f_{\mu}^{-n}(I)$ consists of $2^{n}$ disjoint closed intervals (satisfying $\left.f_{\mu}^{-(n+1)}(I) \subset f_{\mu}^{-n}(I)\right)$, and in the limit we get

$$
\Lambda=\cap_{n=0}^{\infty} f_{\mu}^{-n}(I)=\lim _{n \rightarrow \infty} f_{\mu}^{-n}(I) .
$$

The set $\Lambda$ is closed (as intersection of closed intervals), invariant by construction $\left(\right.$ as $\left.f^{-1}(\Lambda)=f^{-1}\left(\cap_{n=0}^{\infty} f_{\mu}^{-n}(I)\right)=\cap_{n=0}^{\infty} f_{\mu}^{-n}(I)=\Lambda\right)$ and it cannot include any interval (because otherwise, since $f_{\mu}$ is expanding, after finitely many application of $f_{\mu}$ to an interval, we ought to cover the whole set $[0,1])$. Thus $\Lambda$ is a Cantor set.

Moreover, by construction, to any element $x \in \Lambda$ we can associate a symbolic sequence, called Itinerary, or address, of $x$ in the backward 
dynamics, $S_{x}=\left(s_{0} s_{1} s_{2} s_{3} \ldots\right)$ with $s_{i} \in\{0,1\}$, i.e. $S_{x}$ belongs to the set of all one-sided infinite sequences of two symbols $\sum_{2} . S_{x}$ comes from the symbols we put as indices to the intervals in the construction process, and there exists a one-to-one correspondence between the points $x \in \Lambda$ and the elements $S_{x} \in \sum_{2}$. Moreover, from the construction process we have that if $x$ belongs to the interval $I_{s_{0} s_{1} \ldots s_{n}}$ then $f_{\mu}(x)$ belongs to $I_{s_{1} \ldots s_{n}}$. Thus the action of the function $f_{\mu}$ on the points of $\Lambda$ corresponds to the application of the "shift map $\sigma$ " to the itinerary $S_{x}$ in the code space $\sum_{2}$ :

$$
\begin{aligned}
& \text { if } x \in \Lambda \text { has } S_{x}=\left(s_{0} s_{1} s_{2} s_{3} \ldots\right) \\
& \quad \text { then } \\
& f_{\mu}(x) \in \Lambda \text { has } S_{f(x)}=\left(s_{1} s_{2} s_{3} \ldots\right)=\sigma\left(s_{0} s_{1} s_{2} s_{3} \ldots\right)=\sigma\left(S_{x}\right) .
\end{aligned}
$$

Given a point $x \in \Lambda$ how do we construct its itinerary $S_{x}$ ? In the obvious way: we put $s_{0}=0$ if $x \in I_{0}$ or $s_{0}=1$ if $x \in I_{1}$, then we consider $f_{\mu}(x)$ and we put $s_{1}=0$ if $f_{\mu}(x) \in I_{0}$ or $s_{1}=1$ if $f_{\mu}(x) \in I_{1}$, and so on. It is easy to see that each periodic sequence of symbols of period $k$ represents a periodic orbit with $k$ distinct points, and thus a so-called $k$-cycle. Since the elements of $\sum_{2}$ can be put in one-to-one correspondence with the real numbers ${ }^{9}$, we have that the periodic sequences are dense in the space, thus the periodic orbits are dense in $\Lambda$. Also there are infinitely many aperiodic sequences (i.e. trajectories) which are dense in $\Lambda$ and we also have sensitivity with respect to the initial conditions. It follows that the backward dynamics $f_{\mu}$ is chaotic in $\Lambda$. Moreover, we also have nice properties of the forward process we are interested in: for any initial state in $I$, whichever is the forward trajectory, it tends to $\Lambda$ (i.e. the distance of the forward iterated points from $\Lambda$ tends to zero). The Cantor set $\Lambda$ describes all possible forward trajectories.

The construction process with the two contraction functions in (20) leading to the Cantor set in (21) can be repeated with any number of contraction functions defined in a complete metric space $D$ of any dimension, as is well known since the pioneering work by Barnsley. Let us recall the definition of an IFS:

Definition. An Iterated Function System (IFS) $\left\{D ; H_{1}, \ldots H_{m}\right\}$ is a collection of $m$ mappings $H_{i}$ of $D$ into itself.

We can so define $W=H_{1} \cup \ldots \cup H_{m}$ and for any point or set $X \subseteq D$ we define $W(X)=H_{1}(X) \cup \ldots \cup H_{m}(X)$. The main property of this definition is given in the following theorem:

Theorem (see Barnsley 1988, Th.1 p. 82). Let $\left\{D ; H_{1}, \ldots H_{m}\right\}$ be an IFS. If the $H_{i}$ are contraction functions then there exist a "unique

\footnotetext{
${ }^{9}$ We can think for example of the representation of the numbers in binary form.
} 
attractor" $\Lambda$ such that $\Lambda=W(\Lambda)$ and $\Lambda=\lim _{n \rightarrow \infty} W^{n}(X)$ for any non-empty set $X \subseteq D$.

In the case described above with the logistic map we have $D=I=$ $[0,1], H_{1}=f_{0}^{-1}, H_{2}=f_{1}^{-1}$, so that $W(X)=f_{\mu}^{-1}(X)=f_{0}^{-1}(X) \cup f_{1}^{-1}(X)$, and the set $\Lambda=\lim _{n \rightarrow \infty} W^{n}(X)=\lim _{n \rightarrow \infty} f_{\mu}^{-n}(X)$ for any $X \subseteq D$ is the set already described above and obtained in (21).

The existence and uniqueness of the set $\Lambda$ is guaranteed by the theorem and it is also true that given any point or set $X \subseteq D$ by applying each time one of the $m$ functions $H_{i}$ we "converge" to the same set $\Lambda$.

In general, if the sets $D_{i}=H_{i}(D) i \in\{1, \ldots, m\}$ are disjoint, we can put the elements of $\Lambda$ in one-to-one correspondence with the elements of the code space on $m$ symbols $\sum_{m}$. The construction is the generalization of the process described above for the two inverses of the logistic functions. Let $U_{0}=D$ and define

$$
\begin{aligned}
U_{1}= & W\left(U_{0}\right)=D_{1} \cup \ldots \cup D_{m}=H_{1}(D) \cup \ldots \cup H_{m}(D) \subset U_{0} \\
U_{2}= & W\left(U_{1}\right)=W^{2}\left(U_{0}\right)=D_{11} \cup \ldots \cup D_{m m}=H_{1}\left(U_{1}\right) \cup \ldots \cup H_{m}\left(U_{1}\right) \subset U_{1} \\
& \ldots \\
U_{n}= & W\left(U_{n-1}\right)=W^{n}\left(U_{0}\right) \subset U_{n-1}
\end{aligned}
$$

i.e. all the disjoint sets of $U_{1}$ are identified with one symbol belonging to $\{1, \ldots, m\}$, all the disjoint sets of $U_{2}$ are identified with two symbols belonging to $\{1, \ldots, m\}$ ( $m^{2}$ in number) and so on, all the disjoint sets of $U_{n}$ are identified with $n$ symbols belonging to $\{1, \ldots, m\}$ ( $m^{n}$ in number), and in the limit, as $\Lambda=\lim _{n \rightarrow \infty} U_{n}=\lim _{n \rightarrow \infty} W^{n}\left(U_{0}\right)=\cap_{n=0}^{\infty} W^{n}\left(U_{0}\right)$. Each element $x \in \Lambda$ is in one-to-one correspondence with the elements $S_{x} \in \sum_{m}$, where $S_{x}=\left(s_{0} s_{1} s_{2} s_{3} \ldots\right), s_{i} \in\{1, \ldots, m\}$.

Moreover, for any element $x \in \Lambda$ we can define a transformation (or map) $F$ on the elements of $\Lambda$ by using the inverses of the functions $H_{i}$ (the so called shift transformation or shift dynamical system in Barsnley 1988, p. 144):

$$
\text { if } x \in H_{i}(D) \quad \text { then } \quad F(x)=H_{i}^{-1}(D),
$$

so that we can also associate an induced dynamic to the points belonging to $\Lambda$, and the rule described in (22) holds for $F$, i.e. if $x \in \Lambda$ has $S_{x}=$ $\left(s_{0} s_{1} s_{2} s_{3} \ldots\right)$ then $F(x) \in \Lambda$ has $S_{F(x)}=\left(s_{1} s_{2} s_{3} \ldots\right)=\sigma\left(s_{0} s_{1} s_{2} s_{3} \ldots\right)=$ $\sigma\left(S_{x}\right)$. Clearly, when the functions $H_{i}$ are distinct inverses of a unique function $f$ then the induced dynamic system is the same, as $F=f$.

\subsection{The chaos game}

As a second relevant example let us consider another well known IFS with three functions, the so-called chaos game. Choose three different 


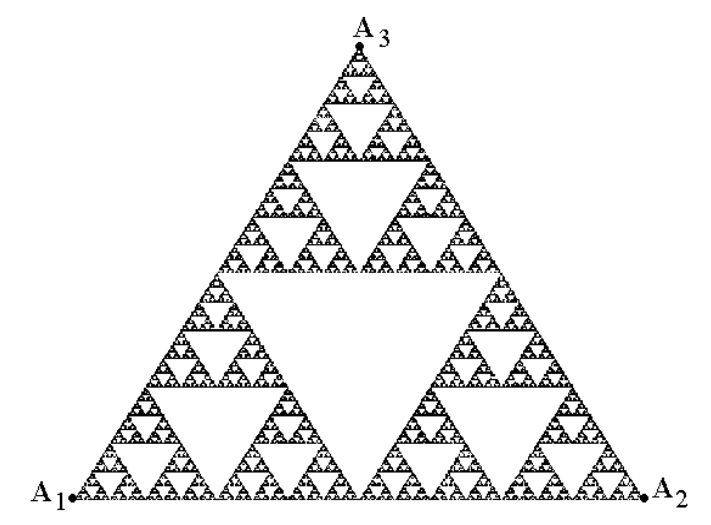

Figure 3: Sierpinski triangle, unique attractor of the IFS $\left\{D ; H_{1}, H_{2}, H_{3}\right\}$.

points $A_{i}, i=1,2,3$, in the plane, not lying on a straight line. Let $D$ be the closed set bounded by the triangle with vertices given by the three points $A_{i}$, and consider the IFS $\left\{D ; H_{1}, H_{2}, H_{3}\right\}$ where the $H_{i}$ are linear contractions in $D$ with center $A_{i}$ and contractivity factor 0.49 . Then choose an arbitrary initial state $x_{0}$ in $D$. An orbit of the system is obtained by applying one of the three maps $H_{i}$, after throwing a dice. More precisely, $x_{n+1}=H_{i}\left(x_{n}\right)$ with $i=1$ after throwing 1 or $2, i=2$ after throwing 3 or $4, i=3$ after throwing 5 or 6 . For any initial state $x_{0} \in D$, plotting points of this orbit after a short transient gives Figure 3 . This fractal shape is called the Sierpinski triangle and it is the unique attractor of the chaos game. Almost all the orbits generated in the chaos game are dense in the Sierpinski triangle.

Moreover, in Barnsley (1988, p. 335) it is also shown how, besides the standard IFS, we can consider a Random IFS (RIFS for short, or IFS with probabilities) by associating a probability $p_{i}>0$ to each function $H_{i}$, such that $\sum_{i=1}^{m} p_{i}=1$. Considering a point $x_{0} \in D$ then we choose recursively

$$
x_{n+1} \in\left\{H_{1}\left(x_{n}\right), \ldots, H_{m}\left(x_{n}\right)\right\}
$$

and the probability of the event $x_{n+1}=H_{i}\left(x_{n}\right)$ is $p_{i}$. The iterated points always converge to the unique attractor $\Lambda$ of the standard IFS, but the density of the points over the set $\Lambda$ reflects in some way the chosen probabilities $p_{i}$. However, we note that if the probabilities in the RIFS are strictly positive, $p_{i}>0$, then the unique attractor does not change, and the iterated points are dense in $\Lambda .{ }^{10}$

\footnotetext{
${ }^{10}$ The reader can find an excellent review on random dynamical systems
} 
This may be very useful and convenient when using IFS theory applied to dynamic economic equilibrium models (see e.g. Montrucchio and Privileggi, 1999, Mitra et al., 2004 and Mitra and Privileggi, 2009 for some economic applications). Using an approach similar to the Random IFS, we can define a Restricted IFS (or IFS with restrictions) imposing that, depending on the position of a point $x \in D$ not all the maps $H_{i}$ can be applied but only some of them. Stated differently we can impose some restrictions on the order in which the functions can be applied. As an example let us consider the chaos game described above, but now with some restrictions, that is, the order in which the three different maps $H_{i}$ are applied is not completely random, but subject to certain restrictions. Suppose for example that the map $H_{1}$ is never applied twice consecutively, i.e. whenever $H_{1}$ is applied then the next map to be applied is either $H_{2}$ or $H_{3}$. Let $\sum^{*} \subset \sum_{3}$ (the code space on three symbols) be the subset of all sequences which do not have two consecutive 1's. The chaos game $\left\{D ; H_{1}, H_{2}, H_{3}\right\}$ with the restriction so described has a unique attractor $\Lambda^{*}$ whose points are in one-to-one correspondence with the restricted space $\sum^{*}$. A typical orbit of this chaos game with restrictions, after a short transient, is shown in Figure 4. The unique attractor of the chaos game with restrictions is a subset of the Sierpinski triangle, the attractor of the chaos game. In fact, the attractor contains precisely those points of the Sierpinski triangle whose itinerary, or addresses, do not have two consecutive 1's.

This example shows that when some restrictions upon the order in which the maps are applied is imposed, then a unique fractal attractor can arise, which is some subset of the unique attractor of the IFS.

In the following sections we shall see how IFS are related in a natural way to non-uniquely defined forward equilibrium dynamics. We will also see that the forward dynamics can be described by an IFS, whenever the (uniquely defined) backward dynamics has homoclinic trajectories due to the existence of a snap-back repellor.

\section{Analysis of the 1-D OLG-model}

Let us consider the one-dimensional OLG model described in Subsection 2.1. Recall that the backward dynamics is given by

$$
y_{t}=\chi\left(y_{t+1}\right)
$$

Whenever the graph of the offer curve displays a hump then for a given $y_{t}$ there are two equilibrium values of future output $y_{t+1}$. Define the part that lies to the left of the critical point $y_{m}$ of $\chi$ as the left map

in Bhattacharya and Majumdar (2004). 


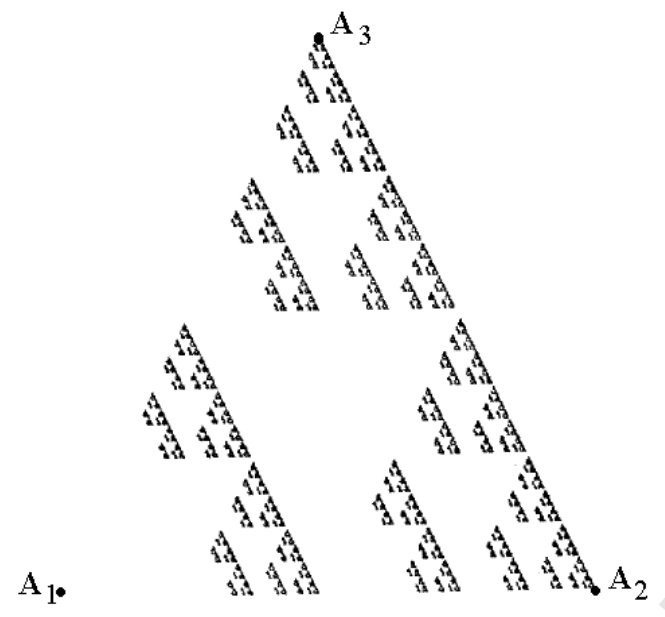

Figure 4: A subset of the Sierpinski triangle. $\Lambda^{*}$ is the unique attractor of the RIFS $\left\{D ; H_{1}, H_{2}, H_{3}\right\}$ with the restriction that whenever $H_{1}$ is applied then it is followed by either $\mathrm{H}_{2}$ or $\mathrm{H}_{3}$.

and the part that lies to the right of $y_{m}$ as the right map. In this way we obtain two invertible functions, one monotonically increasing and the other monotonically decreasing. Call the inverse of the left map $\chi_{L}^{-1}$ and the inverse of the right map $\chi_{R}^{-1}$ (see Fig.5). Future output is then given by either the left inverse map

$$
y_{t+1}=\chi_{L}^{-1}\left(y_{t}\right)
$$

or by the right inverse map

$$
y_{t+1}=\chi_{R}^{-1}\left(y_{t}\right) .
$$

At this point it is useful to consider a concrete specification of the 1-D OLG-model. As in Grandmont (1985), let

$$
V_{1}\left(l^{*}-l\right)=\frac{\left(l^{*}-l\right)^{1-\alpha_{1}}}{1-\alpha_{1}} \quad \text { and } \quad V_{2}(c)=\frac{(0.5+c)^{1-\alpha_{2}}}{1-\alpha_{2}},
$$

with $l^{*}=2, \alpha_{1}=0.5$ and $\alpha_{2}>0$. Figure 5 shows the shape of $\chi$.

Except for the steady state at the origin, asymptotically the backward dynamics (23) is contained in the invariant interval $I=\left[\chi^{2}\left(y_{m}\right), \chi\left(y_{m}\right)\right]$, where $y_{m}$ is the critical point. Notice that for any point outside this interval $I$, the forward process either tends to zero (after applying the left inverse map (24) infinitely many times) or is undefined when it enters a region without preimages (after applying the right inverse map (25) once). We therefore restrict attention to the interval $I$. 


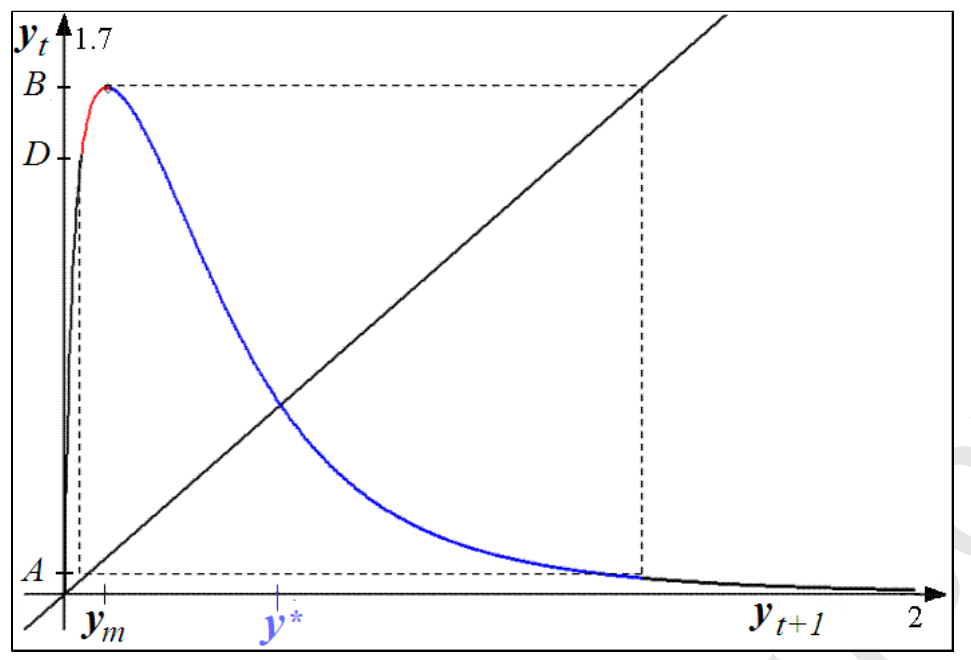

Figure 5: Graph of the one-dimensional function $\chi$ at $l^{*}=2, \alpha_{1}=0.5$ and $\alpha_{2}=6 . \chi_{L}$ is the branch on the left of the critical point $y_{m}$ with inverse $\chi_{L}^{-1}, \chi_{R}$ is the branch on the right of $y_{m}$ with inverse $\chi_{R}^{-1}$. The interval of interest is $I=[A, B]=[A, D] \cup[D, B]$, where $B=\chi\left(y_{m}\right)$, $A=\chi^{2}\left(y_{m}\right), D=\chi^{3}\left(y_{m}\right)$.

Moreover, the third iterate $\chi^{3}\left(y_{m}\right)$ of the critical point $y_{m}$ separates the interval $I$ into two parts (see Fig.5). For any point belonging to the interval $\left[\chi^{2}\left(y_{m}\right), \chi^{3}\left(y_{m}\right)\right]$ only the right inverse, $\chi_{R}^{-1}$, leads to an interesting forward trajectory, as application of the left inverse leads out of $I$. On the other hand, whenever we have a point $y$ in the interval $\left[\chi^{3}\left(y_{m}\right), \chi\left(y_{m}\right)\right]$ then both the inverses $\chi_{L}^{-1}$ and $\chi_{R}^{-1}$ for the forward process can be applied giving again a point in $I$ (and clearly $\chi_{L}^{-1}(y) \leq y_{m}$ , $\left.\chi_{R}^{-1}(y) \geq y_{m}\right)$.

Now consider the backward dynamics (23). Figure 6 shows a bifurcation diagram of the backward dynamics. The diagram shows the long run backward dynamics as a function of $\alpha_{2} \in[1,20]$. The backward dynamics exhibits the well-known period-doubling bifurcation route to chaos (Grandmont, 1985).

For continuous 1-D maps $f$ on the interval it is important to know conditions under which the dynamics is chaotic (in the sense of Devaney, 1986). The condition of "existence of an orbit of period 3" is now very well known, since the celebrated work by Li and Yorke (1975). That is, this is a sufficient condition to establish the existence of an invariant (repelling) Cantor set (which may be of zero measure in $I$ ) on which $f$ is chaotic.

Another useful condition is given in Mitra (2001, p. 141-142): $f^{3}\left(x_{m}\right)<$ 


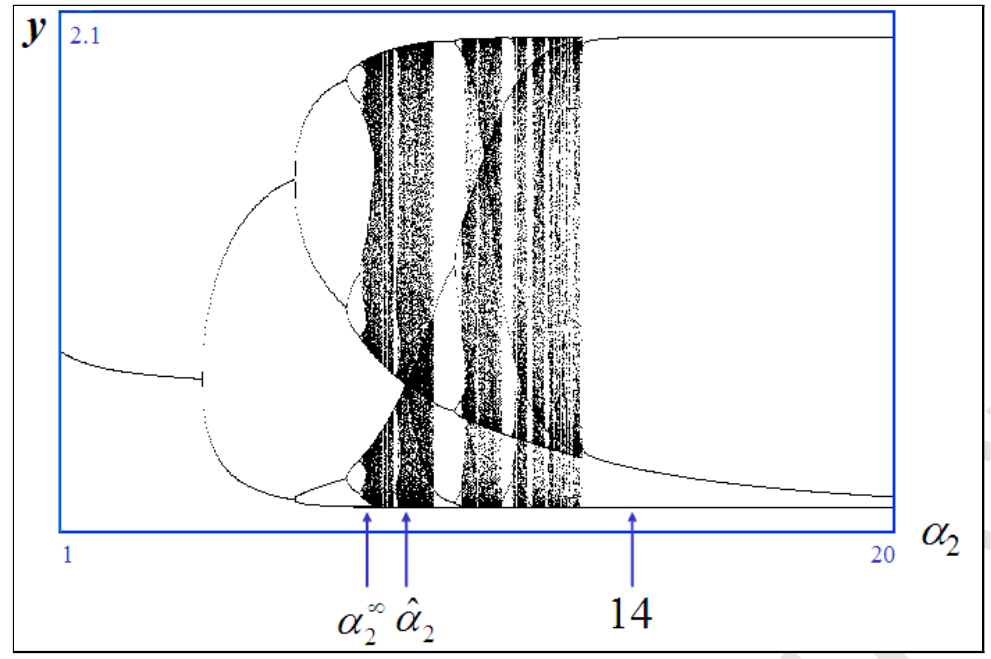

Figure 6: Bifurcation diagram of the function $\chi$ at $l^{*}=2, \alpha_{1}=0.5$ fixed, and varying $\alpha_{2}$ in the interval $[1,20] . \alpha_{2}^{\infty}$ denotes the limit point of the first period doubling cascade. $\widehat{\alpha}_{2}$ denotes the bifurcation value at which $\chi^{3}\left(y_{m}\right)=y^{*}$.

$x^{*}$, where $x_{m}$ denotes the critical point and $x^{*}$ the fixed point. This is exactly the condition for which the fixed point has homoclinic orbits (or the fixed point is a snap-back repellor). An orbit is homoclinic to a fixed point if a sequence of primages from the fixed points tends to the fixed points itself; the notion of homoclinic orbit dates back to Poincaré. A fixed point $x^{*}$ of a map $f$ is called a snap-back repellor of $f$ if all the eigenvalues of $D f\left(x^{*}\right)$ are greater than 1 in modulus, and there exists a (noncritical) homoclinic orbit of $x^{*}$ (Marotto 1978, 2005).

In Figure 6 the critical parameter is denoted by $\widehat{\alpha}_{2}$, that is, for $\widehat{\alpha}_{2}$ (when 2 chaotic intervals merge into a unique one) we have $\chi^{3}\left(y_{m}\right)=$ $y^{*}$, and the backward dynamics are chaotic in the interval $I$ defined above. A (noncritical) homoclinic orbit implies topological chaos (see e.g. Devaney, 1986, Th. 16.5, p. 123). A different proof is in Gardini (1994, Th.1), from which it follows that the condition given in Mitra (2001) can be enforced (emphasizing when the first homoclinic orbits appear) by stating the following:

Proposition 1. Let $x_{m}$ be the maximum point of a unimodal continuous map of an interval into itself, say $f: I \rightarrow I$, smooth in $I \backslash\left\{x_{m}\right\}$, with a unique unstable fixed point $x^{*}$, and a sequence of preimages of $x_{m}$ tends to $x^{*}$. Then the first homoclinic orbits (all critical) of the fixed point $x^{*}$ occur when the critical point satisfies $f^{3}\left(x_{m}\right)=x^{*}$. For $f^{3}\left(x_{m}\right)<x^{*}$ the fixed point is a snap-back repellor. There exists a closed invariant set 
$\Lambda \subseteq\left[f^{2}\left(x_{m}\right), f\left(x_{m}\right)\right] \subseteq I$ on which the map is topologically conjugate to the shift automorphism, and thus $f$ is chaotic, in the sense of Devaney, i.e. topological chaos, with positive topological entropy.

Clearly the proposition holds also for a critical point minimum, with obvious changes. The proof of the bifurcation condition is immediate, as for $f^{3}\left(x_{m}\right)>x^{*}$ the fixed point $x^{*}$ has no rank-1 preimages in $I$, while at $f^{3}\left(x_{m}\right)=x^{*}$ the critical point is homoclinic and infinitely many homoclinic trajectories exist, all critical. When $f^{3}\left(x_{m}\right)<x^{*}$ then infinitely many noncritical homoclinic orbits exist (close to those critical at the bifurcation value, that is, the homoclinic points are obtained by the same sequences of preimages of the function). A technique to construct a Cantor set $\Lambda$ associated with an homoclinic orbit, as well as the related chaotic dynamics, will be discussed in the following.

Moreover, the same result (i.e. existence of a closed invariant set $\Lambda$ on which the map is chaotic) holds for any cycle (periodic point of any period), which is a snap-back repellor (i.e. when homoclinic orbits exist), because proposition 1 can be applied to fixed points of the map $f^{k}$, for any $k>1$ (in suitable intervals for $f^{k}$, corresponding to cyclical intervals for $f$ ). It follows that when such a proposition is applied to a fixed point of the map $f$, it can in fact be applied to infinitely many different $k$-cycles. We shall see an example with our OLG model.

In Figure 6 the point denoted by $\alpha_{2}^{\infty}$ represents the limit of the first period doubling sequence, after which the backward dynamics is (topologically) chaotic, and the cycles of period $2^{n}$ become homoclinic, i.e. snap-back repellors, in decreasing order of period.

\section{Main result}

We are now ready to state the main result concerning the relation between the forward dynamics and the backward dynamics of the 1-D OLGmodel:

Theorem 1. When the offer curve satisfies $\chi^{3}\left(y_{m}\right)<y^{*}$ (i.e. the third iterate of the critical point is below the fixed point) then the fixed point is a snapback repeller in the backward dynamics and there exists an IFS $\{U ; F, G\}$ with a unique fractal attractor $\Lambda$, such that

(i) $\Lambda$ is a repelling invariant Cantor set of the backward dynamics;

(ii) $\Lambda$ is the unique attractor of the $\operatorname{IFS}\left(\Lambda=\lim _{n \rightarrow \infty} W^{n}(X)\right.$ for any $X \subseteq U$, where $W=F \cup G$ );

(iii) For any initial state in $U$, the forward dynamics obtained with the functions $F$ and $G$ always stays in $U$ and tends to $\Lambda$ or a subset of $\Lambda)$;

(iv) If the probabilities in the Random IFS are strictly positive, $p_{i}>0$, the forward trajectory obtained with the contraction functions $F$ and $G$ 
is dense in $\Lambda$.

(v) Each orbit of the IFS is a sunspot equilibrium of the OLG-economy.

The condition $\chi^{3}\left(y_{m}\right)<y^{*}$ implies that the fixed point is a snapback repellor for the backward dynamics. The maps $F$ and $G$ are composite maps of the left inverse map $\chi_{L}^{-1}$ in $(24)$ and right inverse map $\chi_{R}^{-1}$ in (25). For example, in the sketch of the proof below, $F=\chi_{L R R R}^{-4}$ and $G=\chi_{R R R R}^{-4}$. The theorem in fact holds more generally, whenever the backward dynamics has an unstable periodic orbit which is a snap-back repellor. This will be the case for all $\alpha_{2}$-values beyond the limit point of the first period doubling cascade (denoted by $\alpha_{2}^{\infty}$ in Figure 6, beyond which cycles of period $2^{n}$ become snapback repellors).

\section{Sketch of the proof (1-D case)}

We sketch the proof of Theorem 1 by first considering a concrete example and then discuss how the construction can be generalized. As an example, fix $\alpha_{2}=14$, at which the backward dynamics of $\chi$ converges to a 3 -cycle.

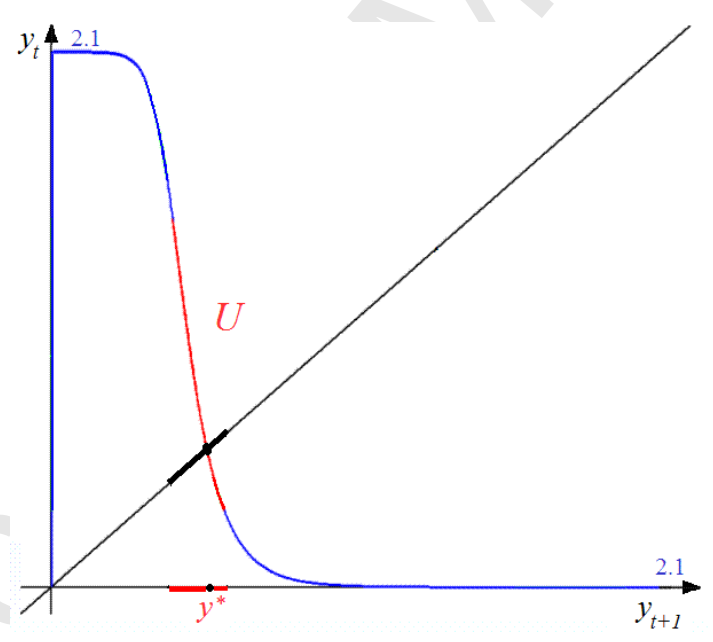

Figure 7: Starting set U, neighborhood of $y^{*}$ (in which $\left|\chi^{\prime}(x)\right|>1$, for all $x \in U$ ) of the function $\chi$ at $l^{*}=2, \alpha_{1}=0.5$ and $\alpha_{2}=14$.

Consider a set of initial conditions $U$, containing the fixed point $y^{*}$, and such that $\left|\chi^{\prime}(x)\right|>1$, for all $x \in U$, so that $\chi_{R}^{-1}(y)$ is a contraction in $U$ (see Figure 7 ). Now apply to $U$ the left inverse map $\chi_{L}^{-1}$ one time, and then the right inverse map $\left(\chi_{R}^{-1}\right) n$ times, where $n$ is such that the final set is again located inside $U$. Such an integer $n$ exists because the fixed point is a snap-back repellor and we are following a homoclinic trajectory. Moreover, $\chi_{R}^{-1}$ is a decreasing function having no periodic 
points to the right of $y_{m}$ except for the fixed point $y^{*}$, thus applying the right inverse map repeatedly to a point or an interval, it will converge to the fixed point. In our example we need three consecutive applications of $\chi_{R}^{-1}$ to obtain a set $I_{0}$ such that $I_{0} \subset U$ (see Figure 8). In this way we have built a suitable inverse function $F=\chi_{L R R R}^{-4}$. Another suitable inverse, which we call $G$, can be obtained applying 4 times (as $F$ is formed by 4 composite mappings) the right inverse map $\chi_{R}^{-1}$. The final set $I_{1}=G(U)=\chi_{R}^{-4}(U)$ still contains the fixed point and is such that $I_{1} \subset U$ because $\chi_{R}^{-1}$ is a contraction. Clearly $I_{1}$ and $I_{0}$ are disjoint (because the first iterates $\chi_{R}^{-1}(U)$ and $\chi_{L}^{-1}(U)$ are disjoint, and then we apply only the function $\left.\chi_{R}^{-1}\right)$.

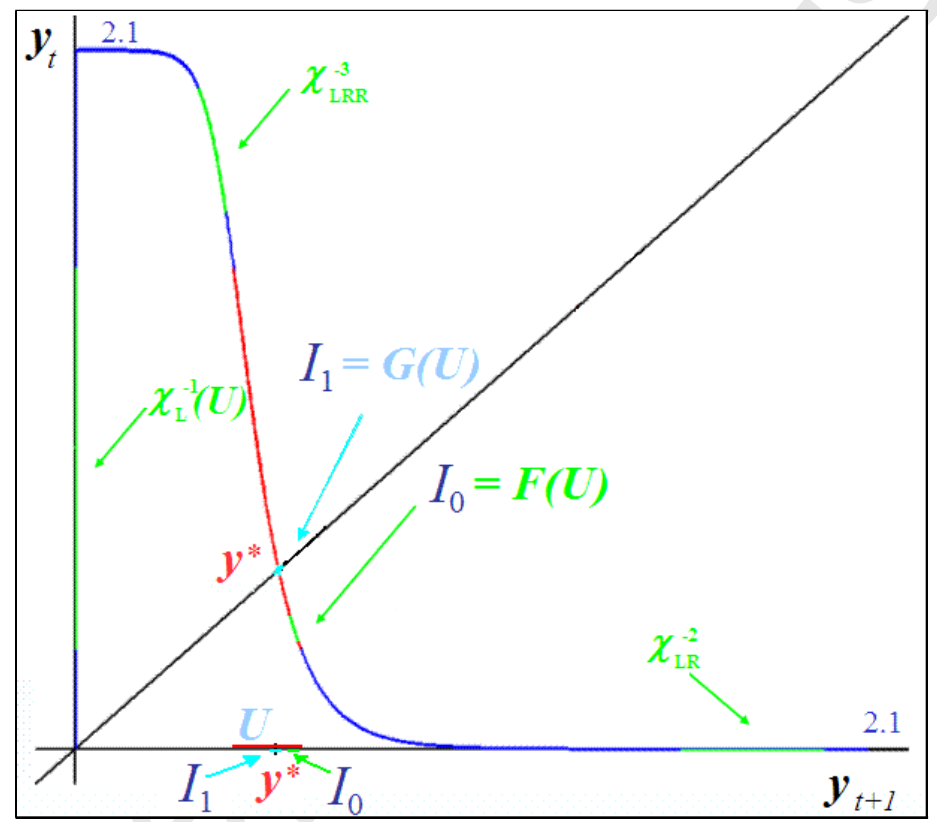

Figure 8: A building up of the two disjoint intervals $I_{0}$ and $I_{1}$ belonging to $U$, for the function $\chi$ at $l^{*}=2, \alpha_{1}=0.5$ and $\alpha_{2}=14 . y^{*} \in I_{1}$.

Thus, by construction we have $I_{0} \cap I_{1}=\varnothing, \chi^{4}\left(I_{1}\right) \supset\left(I_{0} \cup I_{1}\right)$ and $\chi^{4}\left(I_{0}\right) \supset\left(I_{0} \cup I_{1}\right)$ (as $\left.\chi^{4}\left(I_{1}\right)=\chi^{4}\left(I_{0}\right)=U\right)$, and these are sufficient conditions to apply the standard technique used in symbolic dynamics and in the Iterated Function System (IFS) theory (see Barnsley, 1988). Let $U_{0}=U$ and

$$
U_{1}=I_{0} \cup I_{1}=F(U) \cup G(U) \subset U
$$

then we use the two suitable inverses $F$ and $G$. $F\left(U_{1}\right)$ is made up of two disjoint pieces $I_{00}$ and $I_{01}$ belonging to $I_{0}$, such that $\chi^{4}\left(I_{00}\right)=I_{0}$ and 
$\chi^{4}\left(I_{01}\right)=I_{1}$. Similarly $G\left(U_{1}\right)$ is made up of two disjoint pieces $I_{10}$ and $I_{11}$ belonging to $I_{1}$, and $\chi^{4}\left(I_{10}\right)=I_{0}$ and $\chi^{4}\left(I_{11}\right)=I_{1}$, and so on. By repeating iteratively this mechanism we construct a nested sequence of closed sets $U_{n+1}=F\left(U_{n}\right) \cup G\left(U_{n}\right) \subset U_{n}$ (for $n \geq 1$ each $U_{n}$ consists of $2^{n}$ intervals) and in the limit process we get a closed invariant set $\Lambda \subset U$, whose elements are in one-to-one correspondence with the elements of the space $\sum_{2}$ of one sided infinite sequences on two symbols $\{0,1\}$ and the application of the map $\chi^{4}$ to an element of $\Lambda$ corresponds to the application of the shift map $\sigma$ to an element of $\sum_{2}$. We have

$$
\Lambda=\bigcap_{n>0} U_{n}=\lim _{n \rightarrow \infty} U_{n} \quad, \quad U_{n+1}=F\left(U_{n}\right) \cup G\left(U_{n}\right) \subset U_{n}
$$

When $F$ and $G$ are contractions, then $\Lambda$ is a classical Cantor set of points on which the map $\chi^{4}$ is chaotic, and the condition on the first derivative $\left|\left(\chi^{4}\right)^{\prime}(x)\right|>1$ in any point $x \in U$ is enough to state that the two inverses $F$ and $G$ are contractions. Clearly $G$ is a contraction by construction (composition of a finite number of contracions), while we have to prove this for $F$. The easiest way is to consider the minimum absolute value of the derivate $\left(\chi^{\prime}\right)$ in the intervals $U, \chi_{L}^{-1}(U), \chi_{L R}^{-2}(U)$, $\chi_{L R R}^{-3}(U)\left(\right.$ as $\left.I_{0}=\chi_{L R R R}^{-4}(U) \subset U\right)$, which we can call $\xi_{i}^{\min }$ for $i=1$ to 4 , respectively, and check if $\prod_{i=1}^{4} \xi_{i}^{\mathrm{min}}$ is higher than 1 . In our case this condition holds. If not, we have to consider more applications of $\chi_{R}^{-1}$, and we are sure (as the derivative $\chi_{R}^{\prime}$ is higher than 1 in $U$ ) that an integer $k$ exists such that the condition on the derivative is satisfied also by the function $F=\chi_{L R \ldots R}^{-k}$ in $U$. Thus both $F$ and $G$ are contractions on the starting set $U$. Then $\{U ; F, G\}$ forms an IFS. That is, in $U$ we can define the set valued function $W()=.F(.) \cup G($.$) , and \Lambda$ is the unique attractor of the IFS:

$$
W(\Lambda)=\Lambda \text { and } \Lambda=\lim _{n \rightarrow \infty} W^{n}(X) \text { for any } X \subseteq U .
$$

This proves properties (i)-(iii) of Theorem 1 for the case $\alpha_{2}=14$. Property (iv) follows immediately from the results on RIFS discussed in Subsection 3.2, while property (v) follows from the general discussion of sunspot equilibria in Subsection 2.3.

The same construction can be applied for any value $\alpha_{2}>\widehat{\alpha}_{2}$, that is, when the fixed point is a snap-back repellor. Moreover, a similar construction applies to any $\alpha_{2}>\alpha_{2}^{\infty}$, i.e. for parameter values beyond the accumulation point of period doubling bifurcations. For all these parameter values there exits some cycle with homoclinic orbits, that is, some cycle is a snap-back repellor, and we can apply a similar construction.

As another example, consider the case $\alpha_{2}=8.22<\widehat{\alpha}_{2}$, for which the 2 -cycle is a snap-back repellor (i.e. homoclinic orbits to the 2-cycle exist). 


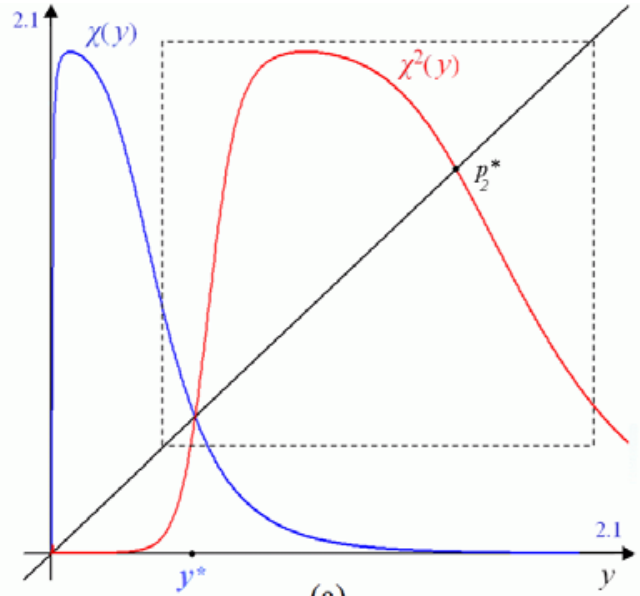

(a)

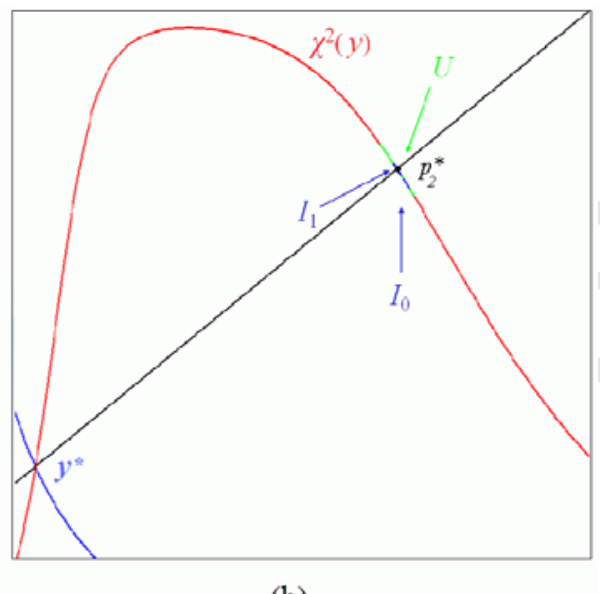

(b)

Figure 9: In (a) graph of the function $\chi$ at $l^{*}=2, \alpha_{1}=0.5$ and $\alpha_{2}=8.22$, $\alpha_{2}^{\infty}<\alpha_{2}<\widehat{\alpha}_{2}$ and the graph of the second iterate of the function, $\chi^{2}$. In (b) an enlargement of $\chi^{2}$ showing a point of the 2-cycle which is a snap-back repellor.

Figure 9 illustrates that on a suitable interval $[a, b]$ around the largest point $p_{2}^{*}$ of the 2 -cycle, for the function $\chi^{2}$ we are in the same situation as before for the fixed point $y^{*}$, with two local inverses which we call $\left(\chi^{2}\right)_{R}^{-1}$ and $\left(\chi^{2}\right)_{L}^{-1}$, and the 2-cycle is a snap-back repellor, thus we can repeat the process. Figure 9(b) shows a suitable neighbourhood $U$ and the two intervals $I_{1}=G(U)=\left(\chi^{2}\right)_{R}^{-k}(U)$ around $p_{2}^{*}$ and $I_{0}=F(U)=$ $\left(\chi^{2}\right)_{L R \ldots R}^{-k}(U) \subset$ for a suitable integer $k$. Then $\{U ; F, G\}$ constitutes an IFS.

Moreover, as discussed in Section 3, we can also consider the IFS with probabilities, or Random Iteration Function System (RIFS) $\left\{U ; F, G ; p_{1}, p_{2}\right\}$, $p_{i}>0, p_{1}+p_{2}=1$, which means that given a point $x \in U$ we consider the trajectory obtained by applying the function $F$ with probability $p_{1}$ or the function $G$ with probability $p_{2}$, that is, one of the functions is selected at random, with the given probabilities. The sequence of points is trapped in $U$, i.e. the forward trajectory cannot escape, and the qualitative shape of the asymptotic orbit has the set $\Lambda$ as a "ghost" underlying it. Some points in $\Lambda$ are visited more often than others, that is, typical forward trajectories may be described by an invariant measure with support on the fractal set $\Lambda$.

It should be clear that once we know some cycle to be a snap-back repellor, then we can construct infinitely many IFS, that is, starting from a fixed neighborhood, with different contractions we can construct as many invariant sets as we want, and in each we have suitable com- 
positions of the two inverses of the map $\chi_{L}^{-1}$ and $\chi_{R}^{-1}$. Ideally we can think of a RIFS in which we put all possible IFS, with some positive probability, in such a way that whenever we apply the function $\chi_{L}^{-1}$ then we apply the function $\chi_{R}^{-1}$ as often as needed. For example, for the RIFS constructed above for $\alpha_{2}=8.22$, whenever the function $\chi_{L}^{-1}$ is applied, thereafter the function $\chi_{R}^{-1}$ is applied at least 7 times, and so on. Thus, roughly speaking, we conclude that "the generic forward trajectory" obtained in this way is a random sequence of points in the bounded region obtained by the starting interval $U$ and its images with the functions which are involved in the definition of the contractions of the IFS. In our example $\alpha_{2}=8.22$, the set including all the forward states includes $U, \chi_{L}^{-1}(U), \chi_{L R}^{-2}(U), \ldots, \chi_{R L \ldots L}^{-(8)}$, that is, the trajectory always belongs to the set

$$
S=U \cup \chi_{L}^{-1}(U) \cup \chi_{L R}^{-2}(U) \cup \ldots \cup \chi_{R L \ldots L}^{-(8)} .
$$

Moreover, it is not always necessary to apply the function $\chi_{L}^{-1}$ only once in a row. In fact IFS may be constructed (especially at high values of $\left.\alpha_{2}\right)$ in which two consecutive applications of $\chi_{L}^{-1}$ can occur. Thus we can conclude that "the generic forward trajectory" (with the only constraint that we cannot apply the function $\chi_{L}^{-1}$ when it leads outside of the absorbing interval $I$ ) is a random sequence of points in the bounded absorbing interval $I$ with points more dense in particular intervals.

\section{Analysis of the 2-D OLG-model}

Recall for the 2-D OLG model that the forward dynamics is defined by

$$
\begin{aligned}
& q_{t+1}=r_{t} \\
& r_{t+1}=\left(1-\delta+\frac{1}{a}\right) r_{t}-\frac{1}{a} \chi^{-1}\left(q_{t}\right),
\end{aligned}
$$

and the backward dynamics is described by the two-dimensional backward map

$$
\left(q_{t}, r_{t}\right)=T\left(q_{t+1}, r_{t+1}\right)=\left(\chi\left[a\left(1-\delta+\frac{1}{a}\right) q_{t+1}-a r_{t+1}\right], q_{t+1}\right) .
$$

If the offer curve $\chi$ is non-monotonic, the backward map $T$ is noninvertible and the forward dynamic is not uniquely defined. The backward map $T$ is of so called ${ }^{11}$ type $Z_{0}-Z_{2}$ (as for the $1 \mathrm{D}$ case): there exists a critical curve in which the determinant of the Jacobian matrix $D T$ vanishes, say $L C_{-1}$, a straight line of equation

$$
r_{t}=\left(1-\delta+\frac{1}{a}\right) q_{t}-\frac{y_{m}}{a},
$$

\footnotetext{
${ }^{11}$ Mira et al. (1996).
} 
where $y_{m}$ is the critical point of the offer curve as before. The critical line plays the same role as the critical point $y_{m}$ in the $1 \mathrm{D}$ case, and separates the phase plane in two regions, denoted by $L$ and $R$, on the left (or upper) part and the right (or lower) part, respectively (see Figure 10). The critical line is mapped onto the line $L C=T\left(L C_{-1}\right)$, a vertical line $q_{t}=\chi\left(y_{m}\right)$, which separates the plane in two regions $Z_{0}$, each point of which has no forward value, and $Z_{2}$, each point of which has two distinct forward values, say $T_{L}^{-1}($.$) and T_{R}^{-1}$ (.) located at opposite sides of the curve $L C_{-1}$, one on the left, $L$, and one on the right, $R$, respectively.

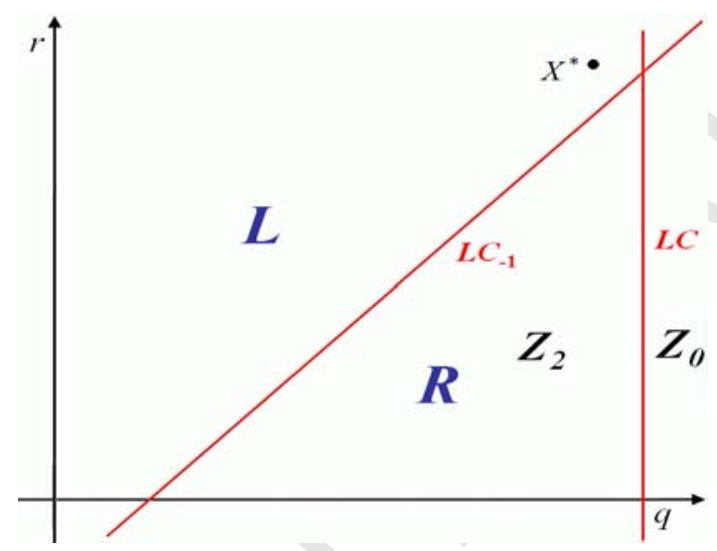

Figure 10: Two-dimensional phase plane separated in two regions $(R$ and $L)$ by the critical line $L C_{-1}$. Each point on the right of the line $L C=T\left(L C_{-1}\right)$, region $Z_{0}$, has no rank-1 preimages, while each point on the left of the line $L C$, region $Z_{2}$, has two distinct rank-1 preimages, one in $R$ and one in $L$.

We claim that for the 2-D model if the fixed point $X^{*}$ of $T$ (different from the origin) is a snap-back repellor for the backward dynamics, then a similar result as in the $1-D$ case holds:

Theorem 2. When the fixed point $X^{*}$ of the backward dynamics $T$ is a snap-back repellor, then there exist several IFS $(\{U ; F, G\},\{U ; \widetilde{F}, \widetilde{G}\}$, $\left.\left\{U ; H_{1}, H_{2}, H_{3}\right\}, \ldots\right)$ or RIFS $\left(\left\{U ; H_{1}, H_{2}, H_{3} ; p_{1}, p_{2}, p_{3}\right\}, \ldots\right)$ each with a unique fractal attractor $\Lambda$, such that

(i) $\Lambda$ is a repelling invariant Cantor set of the backward dynamics;

(ii) $\Lambda$ is the unique attractor of the IFS $\left(\Lambda=\lim _{n \rightarrow \infty} W^{n}(X)\right.$ for any $X \subseteq U$, where $W=F \cup G$ or $\left.W=H_{1} \cup H_{2} \cup H_{3}\right)$;

(iii) For any initial state in $U$, the forward dynamics obtained with the functions of the IFS always stays in $U$ and tends to $\Lambda$ or a subset of $\Lambda$;

(iv) If the probabilities in the RIFS are strictly positive, $p_{i}>0$, the 
forward trajectory obtained with the contraction functions $H_{i}$ is dense in $\Lambda$.

(v) Each orbit of the IFS is a sunspot equilibrium of the OLG-economy.

Before we sketch the proof, it is useful to make two observations. Firstly, the theorem refers to a situation where the unstable fixed point $X^{*}$, which belongs to the region $L$, is unstable for the backward dynamics and more precisely is an unstable focus with homoclinic trajectories (i.e. a snap-back repeller). In order to check whether this is the case, it is enough to consider the point $T_{R}^{-1}\left(X^{*}\right)$, and then $T_{L}^{-m} \circ T_{R}^{-1}\left(X^{*}\right)$ : if for a suitable $m$ this point reaches a neighborhood of $X^{*}$ (so that it tends to $X^{*}$ as $m$ tends to infinity), then the fixed point is homoclinic (otherwise such a point will enter $Z_{0}$ at a finite number $m$ ). Secondly, if $X^{*}$ is homoclinic we can extend to the two-dimensional map the construction already described in the 1-D case, leading to an invariant chaotic Cantor set $\Lambda$, which includes infinitely many unstable cycles of $T$ and is repelling for the backward dynamics T. In fact (see Marotto, 1978, 2005 and also Gardini 1994, Th.2), in any neighbourhood of such a fixed point, it is possible to detect two closed disjoint "balls" (bounded, closed and simply connected sets), $U$ and $V, U \cap V=\varnothing$, such that for a suitable $m$ we have $T^{m}(U) \supset U \cup V$ and $T^{m}(V) \supset U \cup V$, thus for the backward map $T^{m}$ there exists an invariant set $\Lambda \subset U \cup V$.

\section{Sketch of the proof (2-D case)}

We follow a similar strategy as in the 1-D case. We first consider a concrete example, fixing the parameters (as in Grandmont, 1985, 1993) $a=3, l_{1}^{*}=2, \delta=0.2, \alpha_{1}=0.5$ and $\alpha_{2}=2.45$, construct a number of IFS's for this example and then discuss how the construction can be generalized. For these parameter values the steady state $X^{*}$ is repelling, i.e. the two eigenvalues of $D T$ are in modulus larger than $1^{12}$. By assumption, the steady state $X^{*}$ is a snap-back repellor.

Consider a (small) neighborhood $U$ of $X^{*}$. Apply to $U$ the right inverse map $T_{R}^{-1}(U)$ one time, and then the left inverse map $T_{L}^{-1} n$ times, where $n$ is such that the final set is again located inside $U$ (see Figure 11). If $U$ is small enough, we are sure that this integer exists because we are following a homoclinic trajectory (which we know to exist), thus by applying the left inverse map repeatedly the sequence of sets will converge to the fixed point $X^{*}$. In our example we need 7 consecutive

\footnotetext{
${ }^{12}$ For $\delta=0.2$ and $\alpha_{2}=2.45$ the steady state $X^{*}$ has complex eigenvalues, so it is an unstable focus. In the construction below it is important that the steady state is repelling; whether the eigenvalues are real or complex is not important.
} 
applications of $T_{L}^{-1}$ to obtain a set $I_{0}$ such that $I_{0} \subset U$. In this way we have built a suitable inverse function $F=T_{R L \ldots L}^{-8}$. Another suitable inverse, which we call $G$, can be obtained by applying the left inverse map $T_{L}^{-1} 8$ times (since $F$ is also composed of 8 applications). The final set $I_{1} \equiv G(U) \equiv T_{L}^{-8}(U)$ still contains the fixed point and is such that $I_{1} \subset U$ with $I_{1}$ and $I_{0}$ disjoint sets (because the first iterates $T_{R}^{-1}(U)$ and $T_{L}^{-1}(U)$ are disjoint, and thereafter we only apply the function $T_{L}^{-1}$. Thus, by construction we have $I_{0} \cap I_{1}=\varnothing, T^{8}\left(I_{1}\right) \supset\left(I_{0} \cup I_{1}\right)$ and $T^{8}\left(I_{0}\right)$

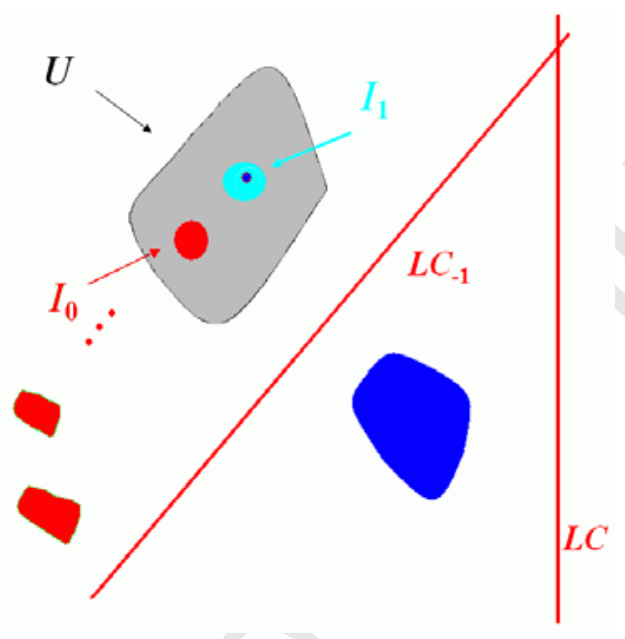

Figure 11: Qualitative description of the neighborhood $U$ involved in the construction of the sets $I_{0}=F(U)=T_{R L \ldots L}^{-8}(U) \subset U$ and $I_{1}=G(U)=$ $T_{L}^{-8}(U) \subset U . X^{*} \in I_{1}$.

$\supset\left(I_{0} \cup I_{1}\right)\left(\right.$ as $\left.T^{8}\left(I_{0}\right)=T^{8}\left(I_{1}\right)=U\right)$, and these are sufficient conditions to apply the construction described in the 1-D case, and find a chaotic invariant set $\Lambda$ for $T^{8}$. Defining $U_{1}=I_{0} \cup I_{1}=F(U) \cup G(U) \subset U\left(=U_{0}\right)$ and iteratively $U_{n+1}=F\left(U_{n}\right) \cup G\left(U_{n}\right) \subset U_{n}$ then in the limit process we get a closed invariant set $\Lambda \subset U$, whose elements are in one-to-one correspondence with the elements of the space $\sum_{2}$ of one sided infinite sequences on two symbols $\{0,1\}$ and the application of the map $T^{8}$ to an element of $\Lambda$ corresponds to the application of the shift map $\sigma$ to an element of $\sum_{2}$. Thus

$$
\Lambda=\bigcap_{n \geq 0} U_{n}=\lim _{n \rightarrow \infty} U_{n}
$$

is the limit set of a nested sequence of closed sets. When $F$ and $G$ are contractions then $\Lambda$ is a classical Cantor set of points, on which the map $T^{8}$ is chaotic. Concerning the properties of the functions $F$ and 
$G$ here constructed, we know that the Jacobian matrix $D G$ has both the eigenvalues in modulus less than 1 in all the points of $U$, and $X^{*}$ is the unique fixed point of $G$ in $U$, belonging to $I_{1}$. This property on the eigenvalues may hold also for the Jacobian matrix $D F$ in all the points of $U$. If not, then we can apply $T_{L}^{-1}$ repeatedly until we have $F=T_{R L \ldots L}^{-(8+m)}$ with this property. This means that also $F$ has a unique fixed point in $U$, belonging to $I_{0}$ (that is, by repeated applications of the function $F$ to $U$ we get a fixed point in $I_{0}$, say $Y_{8}^{*}$ (which is a cycle of period 8 for the backward map $T$ ). As we recall in the Mathematical Appendix, Property 3, the functions $F$ and $G$ are homeomorphic to contractions, and we can always consider suitable neighbourhoods of the related fixed points (by applying $T_{L}^{-1}$ more times repeatedly if necessary), in order for $F=T_{R L \ldots L}^{-(8+m)}$, and the corresponding $G=T_{L L \ldots L}^{-(8+m)}$, to have this property, i.e. two contractions in the Euclidean norm, in a suitable neighborhood $U$. In our example, $F=T_{R L \ldots L}^{-(8)}$ and $G=T_{L L \ldots L}^{-(8)}$ are contractions in the set $U$ shown in Figure 11 (indeed the diameter of $F(U)$ is about half the diameter of $U$, and the same holds for the function $G$ ), so that we have $\bigcap_{n>0} G^{n}(U)=X^{*}, \bigcap_{n>0} F^{n}(U)=Y_{8}^{*}$, and $\Lambda=\bigcap_{n>0} U_{n}=\lim _{n \rightarrow \infty} U_{n}$ is a classical Cantor set of points, that is associated with the IFS $\{U ; F, G\}$.

As for the $1 \mathrm{D}$ case, we have infinitely many choices to construct such functions and related invariant chaotic sets $\Lambda$.

For example, let us construct a second IFS, using two (instead of one) iterations of the right inverse map to the set $U$. That is we consider the neighborhood $U$ of $X^{*}$ given above (i.e. such that the two eigenvalues of $D T$ are in modulus larger than 1 in all the points of $U$ ). Then apply to $U$ the right inverse map $T_{R}^{-1}(U)$ twice, after which the left inverse map $T_{L}^{-1}$ is applied $n$ times, where $n$ is such that the final set is again located inside $U$. Such an integer exists because we are following a homoclinic trajectory (whose existence has been previously verified), thus applying the left inverse map repeatedly the sequence of sets will converge to the fixed point $X^{*}$. In our example we need $k=11$ consecutive applications of $T_{L}^{-1}$ to obtain a set $J_{0}$ such that $J_{0} \subset U$. In this way we have built a suitable inverse function $\widetilde{F}=T_{R R L \ldots L}^{-(2+k)}$, with $k=11$ and we can assume that it is a contraction in the Euclidean norm in $U$ (if not, we adapt $\widetilde{F}$ by appling the left inverse map $T_{L}^{-1}$ as many times as necessary). This means that also $\widetilde{F}$ has a fixed point in $U$, that is, by repeated applications of the function $\widetilde{F}$ to $U$ we get a fixed point in $J_{0}$, say $Z_{(2+k)}^{*}$, $\bigcap_{n>0} \widetilde{F}^{n}(U)=Z_{(k+2)}^{*}$ (which is a cycle of period $(2+k)$ for the backward map $T$ ). Another suitable inverse, which we call $\widetilde{G}$, can be obtained by applying $(2+k)$ times (since $\widetilde{F}$ is also formed by $(2+k)$ iterations) the left 
inverse map $T_{L}^{-1}$. The final set $J_{1}=\widetilde{G}(U)=T_{L}^{-(2+k)}(U)$ still contains the fixed point $X^{*}$ and is such that $J_{1} \subset U$ with $J_{1}$ and $J_{0}$ disjoint sets (because the first two iterates $T_{R R}^{-1}(U)$ and $T_{L L}^{-1}(U)$ are disjoint, thereafter we apply only the function $T_{L}^{-1}$ ), and we can assume that $\widetilde{G}$ is a contraction in $U$ ( since we know that the Jacobian matrix $D \widetilde{G}$ has both the eigenvalues in modulus less than 1 in all the points of $U$ ).

Thus, by construction we have $J_{0} \cap J_{1}=\varnothing, T^{(2+k)}\left(J_{1}\right) \supset\left(J_{0} \cup J_{1}\right)$ and $T^{(2+k)}\left(J_{0}\right) \supset\left(J_{0} \cup J_{1}\right)$ (as $\left.T^{(2+k)}\left(J_{0}\right)=T^{(2+k)}\left(J_{1}\right)=U\right)$, and these are sufficient conditions to apply the mechanism described above, and to construct a Cantor set of points $\widetilde{\Lambda}$ with chaotic structure, invariant for $T^{(2+k)}$, obtained by using the IFS $\{U ; \widetilde{F}, \widetilde{G}\}$, defining $W=\widetilde{F} \cup \widetilde{G}$,

$$
U_{1}=W(U)=J_{0} \cup J_{1}=\widetilde{F}(U) \cup \widetilde{G}(U) \subset U\left(=U_{0}\right)
$$

and iteratively

$$
U_{n+1}=W\left(U_{n}\right)=\widetilde{F}\left(U_{n}\right) \cup \widetilde{G}\left(U_{n}\right) \subset U_{n} .
$$

We get

$$
\widetilde{\Lambda}=\bigcap_{n \geq 0} U_{n}=\bigcap_{n \geq 0} W^{n}(U)=\lim _{n \rightarrow \infty} U_{n}
$$

As a third example, we follow a different approach in order to get in-

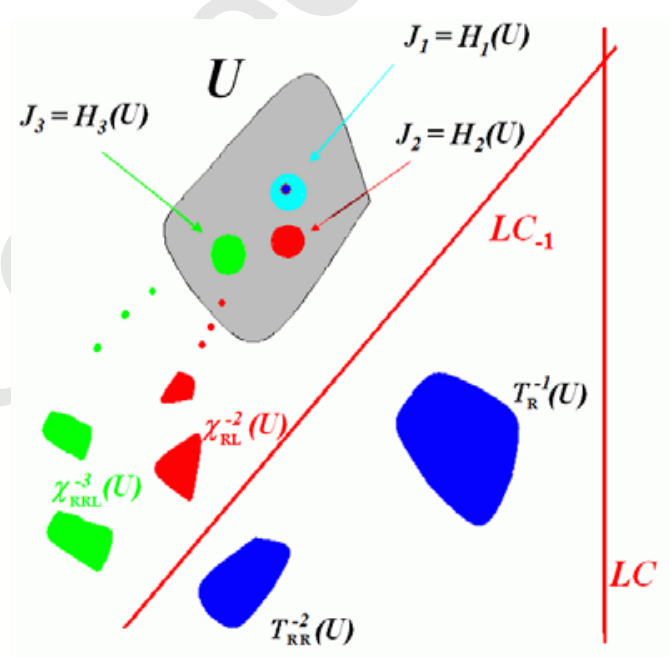

Figure 12: Qualitative description of the construction of the different sets $J_{1}, J_{2}$ and $J_{3}$ belonging to $U$, involved in the IFS similar to the "chaos game" associated with the two-dimensional model. $X^{*} \in J_{1}$.

variant sets, applying the iterated function systems technique to three 
functions. In this way, we will obtain an IFS similar to the chaos game, as discussed in Subsection 3.2, describing forward trajectories.

Consider the following functions: $H_{1}=\widetilde{G}=T_{L}^{-(13)}, H_{2}=\widetilde{F}=$ $T_{R R L \ldots L}^{-(13)}$, and $H_{3}=T_{R L \ldots L}^{-(13)}$ (note that in the definition of $H_{3}$ the inverse $T_{L}^{-1}$ is applied 12 times, while in the definition of $H_{2}$ the inverse $T_{L}^{-1}$ is applied 11 times). As the first two iterates $T_{L L}^{-2}(U), T_{R R}^{-2}(U)$ and $T_{R L}^{-2}(U)$ are disjoint sets, it follows that also $J_{1}=H_{1}(U), J_{2}=H_{2}(U)$, and $J_{3}=H_{3}(U)$ are disjoint sets, all belonging to $U$ (see Figure 12). In our numerical example we have three contraction functions (in general, as stated above, it is always possible to reach this situation by applying the inverse map $T_{L}^{-1}$ as often as necessary). Thus we can consider the set (invariant for the backward dynamics of $T^{(13)}$ ), associated with the IFS $\left\{U ; H_{1}, H_{2}, H_{3}\right\}$, defining $W=H_{1} \cup H_{2} \cup H_{3}$

$$
U_{1}=W(U)=J_{1} \cup J_{2} \cup J_{3}=H_{1}(U) \cup H_{2}(U) \cup H_{3}(U) \subset U\left(=U_{0}\right)
$$

and iteratively

$$
U_{n+1}=H_{1}\left(U_{n}\right) \cup H_{2}\left(U_{n}\right) \cup H_{3}\left(U_{n}\right) \subset U_{n},
$$

for $n \geq 1$ each $U_{n}$ consists of $3^{n}$ elements, and we have

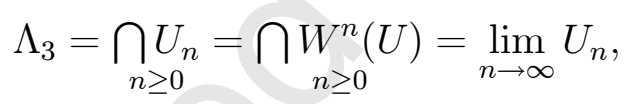

which is an invariant chaotic set (repelling for the backward map T), and the unique attractor in $U$ for the iterated function system.

Moreover, as shown for the 1-D case, we may consider the Random Iteration Function Systems, say RIFS $\left\{U ; H_{1}, H_{2}, H_{3} ; p_{1}, p_{2}, p_{3}\right\}, p_{i}>0$, $p_{1}+p_{2}+p_{3}=1$, which means that given a point $x \in U$ we consider the trajectory obtained by applying the function $H_{i}$ with probability $p_{i}$, that is, at each date one of the functions is selected at random, with the given probabilities. Then the random sequence of points is trapped in $U$, i.e. the forward trajectory cannot escape, and the asymptotic orbit is always dense in the set $\Lambda_{3}$. The distribution of points of the fractal set $\Lambda_{3}$ may be uneven, as some regions may be visited more often than others depending on the magnitude of the probabilities.

It should be clear that the same construction can be applied whenever the fixed point is a snap-back repellor. In fact, whenever some cycle of the backward map is a snap-back repellor, then we can construct infinitely many different IFS, using suitable compositions of the two inverses of the map $T_{L}^{-1}$ and $T_{R}^{-1}$. To describe all forward trajectories, we can ideally think of a RIFS in which we put all suitable compositions of inverses, with some positive probability. To make things concrete, 

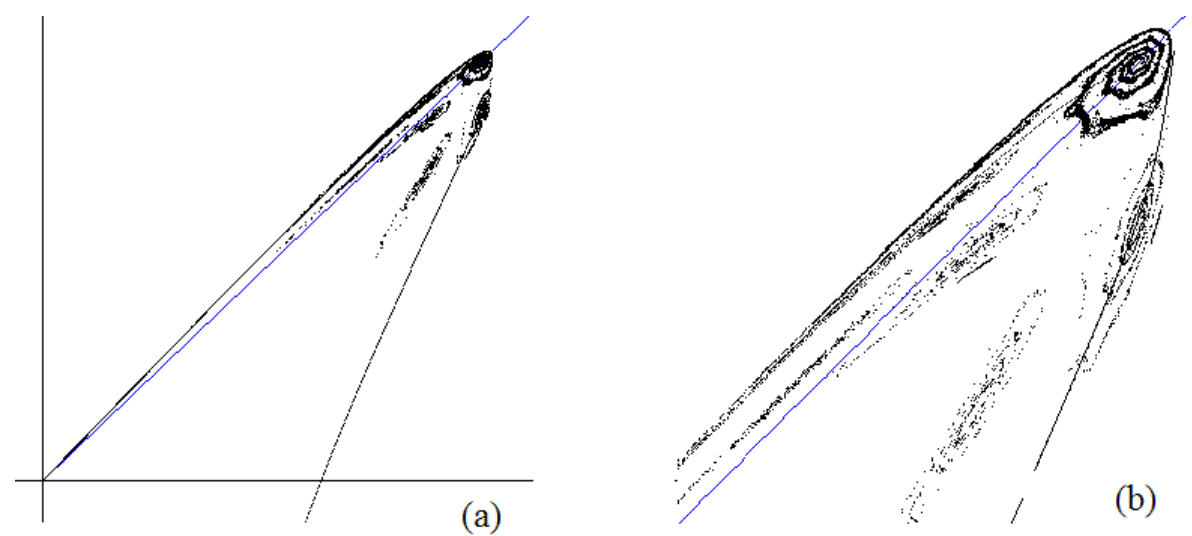

Figure 13: Example of a forward equilibrium trajectory. In (a) a trajectory of the RIFS similar to the "chaos game" associated with the two-dimensional model, at $a=3, l^{*}=2, \delta=0.2, \alpha_{1}=0.5$ and $\alpha_{2}=2.45$. The portion of phase plane $(q, r)$ is $[0.3,0.65] \times[0.3,0.65]$. In (b) an enlargement of (a) is shown.

in the previous example, whenever we apply the function $T_{R}^{-1}$ once or at most two time consecutively, immediately thereafter we must apply the function $T_{L}^{-1}$ as many times as needed, but at least 8 times (if the right $T_{R}^{-1}$ was applied once) or at least 11 times (if the right inverse $T_{R}^{-1}$ was applied twice). Thus, roughly speaking, we can conclude that "the generic forward trajectory" can be obtained as a random sequence of points with an initial state in the set $U$ and its images with the functions which are involved in the definition of the contractions of the IFS. For example, in the chaos game given above the set including all the forward states includes $U, T_{R}^{-1}(U), T_{R L}^{-2}(U), \ldots, T_{R L \ldots L}^{-(13)(U)}, T_{R R}^{-2}(U)$, $T_{R R L}^{-3}(U), \ldots, T_{R R L \ldots L}^{-13}(U)$, that is, the trajectory always belongs to the set

$S=U \cup T_{R}^{-1}(U) \cup T_{R L}^{-2}(U) \cup \ldots \cup T_{R L \ldots L}^{-(13)} \cup T_{R R}^{-2}(U) \cup T_{R R L}^{-3}(U) \cup \ldots \cup T_{R R L \ldots L}^{-13}(U)$

An example of forward trajectory is shown in Fig.13.

\section{Concluding Remarks}

In implicitly defined equilibrium models derived from optimization, the forward dynamics may not be uniquely defined, because at each date there are multiple optimal solutions, while the backward dynamics is well-defined. Well known examples include the overlapping generations model and the cash-in-advance model. Properties of the backward dynamics can be used to describe the forward dynamics. We have applied 
the theory of Iterated Function Systems (IFS) to describe forward equilibrium paths in a one- and a two-dimensional version of the OLG-model. If the backward dynamics has a homoclinic orbit due to a snap-back repellor steady state (or periodic cycle), the forward dynamics can be described by an IFS. The fractal attractor associated with the IFS describes (a large set of) the forward trajectories. Forward trajectories can be obtained by random applications (possibly with suitable restrictions) of the inverse mappings of the backward dynamics. Trajectories of these random IFS can be interpreted as sunspot equilibria, where the random sunspot sequence determines equilibrium selection at each data. The same IFS techniques can be applied to other implicitly defined economic models including higher dimensional systems.

\section{Mathematical Appendix}

This Mathematical Appendix briefly discusses some notions from dynamical systems theory, such as invariant sets, attractors, repellors, homoclinic orbits and snap-back repellors, which play an important role in the relation between forward and backward dynamics of the OLG-model.

Let us consider a $n$-dimensional map with $n \geq 1$,

$$
x^{\prime}=f(x)
$$

where $f: X \rightarrow X$ and the phase space $X$ is a locally compact metric space $X \subset R^{n}$, for $n \geq 1$. A set $A \subseteq X$ is called a trapping set if $f(A) \subseteq A$, it is called invariant for $f$ if $f(A)=A$. The following propery holds:

Property 1. If $A$ is a trapping set for $f$ then any point of $A$ has the whole trajectory in $A$, and $B=\bigcap_{n>0} f^{n}(A)$ is invariant for $f$ (as $\left.f(B)=f\left(\bigcap_{n>0} f^{n}(A)\right)=\bigcap_{n>0} f^{n}(A)=B\right)$.

If $B$ is invariant for $f$ then any point of $B$ has at least one rank-1 preimage in $B$ (as $f(B)=B$ implies that each point of $B$ is the image of at least one point of $B$ ), and iteratively: any point of $B$ has at least an infinite sequence of preimages in $B$.

The behaviour of points in a neighburhood of an invariant set $A$ depends on the local dynamics, A may be attracting, repelling, or neither of the two. Let us recall here some definitions (see Milnor).

An attracting set is a closed invariant set $A$ which possesses a trapping neighborhood, that is, a neighborhood $U$, with $A \subset \operatorname{Int}(U)$, such that:

$$
A=\bigcap_{n \geq 0} f^{n}(U) .
$$


(as in the case of the set $B$ constructed above). In other words, if $A$ is an attracting set for $f$, then a neighborhood $U$ of $A$ exists such that the iterates $f^{n}(x)$ tend to $A$ for any $x \in U$ (and not necessarily enter $A$ ). An attractor is an attracting set with a dense orbit.

A repelling set is a compact invariant set $K$ which possesses an isolating neighborhood; that is a neighborhood $U \supset K$ such that:

$$
K=\bigcap_{n \geq 0}\left(f^{n}\right)^{-1}(U) .
$$

In other words, if $K$ is a repelling set for $f$, then a neighborhood $U$ of $K$ exists such that for any point $x_{0} \in U \backslash K$, the trajectory $x_{0} \rightarrow x_{1} \rightarrow \ldots$ must satisfy $x_{n} \notin U$ for at least one value of $n \geq 0$ (but such a trajectory may also come back again in $U$, as it occurs when homoclinic trajectories exist). A repellor is a repelling set with a dense orbit.

The case of a $k$-cycle $\left\{x_{1}, \ldots, x_{k}\right\}(k \geq 1$, and for $k=1$ it is a fixed point) is studied considering the $k$ fixed points $x_{i}(i=1, \ldots, k)$ of the map $f^{k}$, and if $k>1$ the properties are "cyclical" when related to the map $f$, moreover, as $x_{i+1}=f\left(x_{i}\right)$ we can associate to each point $x_{i}$ of the cycle the local inverse which gives the point $x_{i-1}$, say $f_{i}^{-1}$, (i.e. $\left.f_{i}^{-1}\left(x_{i}\right)=x_{i-1}\right)$ so that each fixed point $x_{i}$ of the map $f^{k}$ has a local inverse, say $\tilde{f}^{-k}$, which is a composition of the local inverses defined on the cycle. For example, in the case of a two cycle $\left\{x_{1}, x_{2}\right\}$, the two points are fixed points of $f^{2}$ and the two local inverses are $\widetilde{f}^{-2}=f_{1}^{-1} \circ f_{2}^{-1}$ and $\tilde{f}^{-2}=f_{2}^{-1} \circ f_{1}^{-1}$, (where $f_{2}^{-1}\left(x_{2}\right)=x_{1}$ and $f_{1}^{-1}\left(x_{1}\right)=x_{2}$ ), and this means that the sequence of inverses $f_{1}^{-1}, f_{2}^{-1}, f_{1}^{-1}, f_{2}^{-1}, \ldots$ gives the same 2 -cycle $\left\{x_{1}, x_{2}\right\}$.

The local stability of a $k$-cycle is that of the $k$ fixed points of $f^{k}$ and comes from the $n$ eigenvalues of the Jacobian matrix $J_{f^{k}}=$ $J_{f}\left(x_{k}\right) \ldots J_{f}\left(x_{1}\right)$. When the condition $\left|\lambda_{j}\right|>1$ holds for at least one of the $n$ eigenvalues of $J_{f^{k}}\left(x_{i}\right)$, the cycle is called "unstable". The condition on all the eigenvalues of the Jacobian matrix $J_{f}\left(x^{*}\right):\left|\lambda_{j}\right|<1$ (resp. $>1$ ) for $j=1, \ldots, n$, is a sufficient condition to conclude that the fixed point is an attractor (resp. repellor), and it is not necessary. The following propery holds (from Hirsh and Smale 1974, pp. 279-281, applied to the fixed points for the map $f^{k}$ ):

Property 2. If a $k$-cycle is an attractor (resp. repellor) for $f$ then it is a repellor (resp. attractor) for the local inverse map $\widetilde{f}^{-k}$.

If $A$ is an attractor for $f$ then a suitable neighborhhod $U$ of $A$ exists such that for any $x \in U \backslash A, f^{m}(x)$ tends to $A$ as $m$ tends to infinity.

If $A$ is a repellor for $f$ then a suitable neighborhhod $U$ of $A$ exists such that for any $x \in U \backslash A$ a suitable sequence of preimages $\tilde{f}^{-m}(x)$ tends to $A$ as $m$ tends to infinity. 
Note that for $n$-dimensional maps with $n \geq 2$ we have to pay attention to the definition of repellor, because the statement given above does not hold when the cycle is unstable but not a repellor, for example a saddle cycle is unstable both for the map and the local inverse, and it is not repelling. In the economic context, from Property 2 we have that a cycle which is an attractor (resp. repellor) in the backward dynamics of $f$ (in our case of $\chi$ or of the two-dim. model) then it is a repellor (resp. attractor) for the local inverse, and thus in some forward process. Note also that it may be expected an unstable cycle in the backward dynamics to be stable in some forward process, and this is true in the one-dimensional case (because when $n=1$ an unstable cycle is always a repellor), while for $n \geq 2$ this is true only when the cycle is a repellor (equivalently: when the cycle is unstable but not a repellor, i.e. of saddle type, then it is not true).

It is worth noticing that in $n$-dimensional maps with $n \geq 2$ a repelling (attracting) cycle does not lead directly to a "local expansion (contraction)" in the euclidean norm, which requires (for the expansion) the existence of a constant $s>1$ such that the inequality $\|f(x)-f(y)\|>s\|x-y\|$ holds for any $x, y, x \neq y$, in a suitable neighborhood of $x^{*}$. However, in Hirsch and Smale (1974), pp. 278-281 it is proved that an attracting fixed point $x^{*}$ is a local contraction in a suitable norm; it is locally invertible, for the local inverse $x^{*}$ is a repellor, and the local inverse is a local expansion in a suitable norm. Thus from a mathematical point of view the above "imperfection" can be overcome because a repelling cyle implies the existence of a suitable norm for which the above inequality holds: $d_{1}(f(x), f(y))>s d_{1}(x, y)$ in a neighborhood $W$ of a repelling cycle, for a suitable norm and related distance $d_{1}$. But all the norms in $R^{n}$ are equivalent. Thus an homeomorphism $h$ exists such that, for any pair of points, $d_{1}(x, y)=d_{2}(h(x), h(y))$, where $d_{2}$ denotes the Euclidean distance. It follows $d_{2}(h \circ f(x), h \circ f(y))>s d_{2}(h(x), h(y))$ and for any $(\xi, \eta) \in U=h(W)$ we have $d_{2}\left(h \circ f \circ h^{-1}(\xi), h \circ f \circ h^{-1}(\eta)\right)>$ $s d_{2}(\xi, \eta)$. That is: in the Euclidean norm the function $f$, in a suitable neighborhood $U$ of a repelling cycle, is locally topologically conjugated with an expansion (and thus, qualitatively, the dynamics are the same). We have so proved the following property:

Property 3. If a $k$-cycle is an attractor (resp. repellor) for $f$ then $f$ is a local contraction (resp. expansion) in a suitable norm, and $f$ is locally topologically conjugated with a contraction (resp. expansion) in the Euclidean norm.

Moreover, for a repelling cycle, an important property is the existence of homoclinic orbits. An orbit is homoclinic to a fixed point if a sequence of preimages from the fixed points tends to the fixed points itself. The 
existence of homoclinic orbits leads to the existence of chaotic dynamics. Following Marotto $(1978,2005)$ we shall call such a fixed point, or cycle, a snap-back repellor. More precisely, a fixed point $x^{*}$ of a map $f$ is called a snap-back repellor of $f$ if all the eigenvalues of $D f\left(x^{*}\right)$ are greater than 1 in modulus, and there exists a noncritical homoclinic orbit of $x^{*}$. A homoclinic orbit is called noncritical when none of its points is a critical point of $f$, where a critical point of $f$ is a point in which the Jacobian matrix has one eigenvalue equal to zero.

As remarked above, the existence of homoclinic orbits (i.e. of snapback repellors) implies the existence of an invariant set in which the map $f$ is chaotic (in the standard definition of Li anf Yorke, or Devaney). And this is a basic element not only for the backward dynamics of our OLG models, but also for the forward dynamics in general, which we are interested in. For example, if the graph of the offer curve displays a large hump such that the backward dynamics has homoclinic trajectories, or a periodic orbit of period three, then a Cantor set exists, repelling for the backward dynamics. The forward dynamics can then be described as an IFS with the same Cantor set as its unique attractor.

\section{References.}

Azariadis, C., Guesnerie, R., 1986. Sunspots and cycles. Review of Economic Studies 53, 725-736.

Barnsley, M.F., 1988. Fractals everywhere, Academic Press, San Diego, California.

Barnsley, M.F., Demko, S., 1985. Iterated function systems and the global construction of fractals. The Proceedings of the Royal Society of London A399, 243-275.

Bhattacharya, R., Majumdar, M., 2004. Random dynamical systems: a review. Economic Theory 23, 13-38.

Boldrin, M., Montrucchio, L., 1986. On the indeterminacy of capital accumulation path. Journal of Economic Theory 40, 26-39.

Cass, D., Shell, C., 1983. Do sunspots matter?. Journal of Political Economy 91, 193-227.

Cazzavillan, G., Lloyd-Braga, T., Pintus, P., 1998. Multiple steady states and endogenous fluctuations with increasing returns to scale in production. Journal of Economic Theory 80, 60-107.

Devaney, R., 1986. An Introduction to Chaotic Dynamical Systems. The Benjamin/Cummings Publishing Co., Menlo Park, California.

Gardini, L., 1994. Homoclinic bifurcations in n-dimensional endomorphisms, due to expanding periodic points. Nonlinear Analysis, Theory, Methods \& Applications 23, 1039-1089.

Grandmont, J.M., 1985. On endogenous competitive business cycles. 
Econometrica 53, 995-1045.

Grandmont, J.M., 1988. Nonlinear difference equations bifurcations and chaos: an introduction. Lecture Notes Stanford University.

Grandmont, J.M., 1993. Expectations driven nonlinear business cycles. Rheinisch-Westfälische Akademie des Wissenschaften 397, 7-32.

Grandmont, J.M., 2008, Nonlinear difference equations, bifurcations and chaos: an introduction. Research in Economics 62, 122-177.

Grandmont, J.M., Laroque, G., 1986. Stability of cycles and expectations. Journal of Economic Theory 40, 138-151.

Grandmont, J.M., Pintus, P., de Vilder, R., 1998. Capital-labor substitution and competitive nonlinear endogenous business cycles. Journal of Economic Theory 80, 14-59.

Hirsch, M., Smale, S., 1974. Differential Equations, Dynamical Systems and Linear Algebra. Academic Press, N.Y.

Kamihigashi, T., 1999. Chaotic dynamics in quasi-static systems: theory and applications. Journal of Mathematical Economics 31, 183214.

Kehoe, T., Levine, D., Mas-Colell, A., Woodford, M., 1991. Gross substitutability in large-square economies. Journal of Economic Theory $54,1-25$.

Kennedy, J.A., Stockman, D.R., 2008. Chaotic equilibria in models with backward dynamics. Journal of Economic Dynamics and Control 32, 939-955.

Li, T., Yorke, J.A., 1975. Period three implies chaos. American Mathematical Monthly 82, 985-992.

Marotto, F., R., 1978. Snap-back repellers imply chaos in $\mathrm{R}^{n}$. Journal of Mathematical Analysis and Applications 63, 199-223.

Marotto, F., R., 2005. On redefining a snap-back repeller. Chaos, Solitons \& Fractals 25, 25-28.

Medio, A., Raines, B., 2007. Backward dynamics in economics. The inverse limit approach. Journal of Economic Dynamics and Control 31, 1633-1671.

Michener, R., Ravikumar, B., 1998. Chaotic dynamics in a cashin-advance economy. Journal of Economic Dynamics and Control 22, $1117-1137$.

Milnor, J., Attractor, Scholarpedia article, p.4862, link: http://www.scholarpedia.org/article

Mira, C., Gardini, L., Barugola, A., Cathala, J.C., 1996. Chaotic dynamics in two-dimensional noninvertible maps. World Scientific, Singapore.

Mitra, T., 2001. A sufficient condition for topological chaos with an application to a model of endogenous growth. Journal of Economic 
Theory 96, 133-152.

Mitra, T., Montrucchio, L. and Privileggi, F., 2004. The nature of the steady state in models of optimal growth under uncertainty. Economic Theory 23, 39-71.

Mitra, T. and Privileggi, F., 2009. On Lipschitz continuity of the iterated function system in a stochastic optimal growth model. Journal of Mathematical Economics 45, 185-198.

Montrucchio, L. and Privileggi, F., 1999. Fractal steady states in stochastic optimal control models. Annals of Operations Research 88, 183-197.

Pintus, P., Sands, D., de Vilder, R., 2000. On the transition from local regular to global irregular fluctuations. Journal of Economic Dynamics and Control 24, 247-272.

Reichlin, P., 1986. Equilibrium cycles in an overlapping generations economy with production. Journal of Economic Theory 40, 89-102.

Sharkovsky A.N., 1964. Coexistence of cycles of a continuous map of a line into itself. Ukrainski Matematycheski Zhurnal 16, 61-71.

Sharkovsky A.N., Kolyada, S.F., Sivak, A.G., Fedorenko, V.V., 1997. Dynamic of One-Dimensional Maps. Kluwer Academic Publishers, London.

de Vilder, R., 1995. Endogenous Business Cycles. PhD-thesis Tinbergen Institute Research Series No. 96.

de Vilder, R., 1996. Complicated endogenous business cycles under gross substitutability. Journal of Economic Theory 71, 416-442.

Woodford, M., 1984, Learning to believe in sunspots. Econometrica 58, 287-307.

Woodford, M., 1986. Stationary sunspot equilibria in a finance constrained economy. Journal of Economic Theory 40, 128-137.

Woodford, M., 1994. Monetary policy and price level determinacy in a cash-in-advance economy. Economic Theory 4, 345-380. 